\title{
Modeling Mid-Infrared Diagnostics of Obscured Quasars and Starbursts
}

\section{Citation}

Snyder, Gregory F., Christopher C. Hayward, Anna Sajina, Patrik Jonsson, Thomas J. Cox, Lars Hernquist, Philip F. Hopkins, and Lin Yan. 2013. "Modeling Mid-Infrared Diagnostics of Obscured Quasars and Starbursts." The Astrophysical Journal 768 (2) (April 25): 168. doi:10.1088/0004-637x/768/2/168.

\section{Published Version}

doi:10.1088/0004-637X/768/2/168

\section{Permanent link}

http://nrs.harvard.edu/urn-3:HUL.InstRepos:34353276

\section{Terms of Use}

This article was downloaded from Harvard University's DASH repository, and is made available under the terms and conditions applicable to Other Posted Material, as set forth at http:// nrs.harvard.edu/urn-3:HUL.InstRepos:dash.current.terms-of-use\#LAA

\section{Share Your Story}

The Harvard community has made this article openly available.

Please share how this access benefits you. Submit a story.

\section{Accessibility}




\title{
MODELING MID-INFRARED DIAGNOSTICS OF OBSCURED QUASARS AND STARBURSTS
}

\author{
Gregory F. Snyder ${ }^{1}$, Christopher C. Hayward ${ }^{2}$, Anna Sajina ${ }^{3}$, Patrik Jonsson ${ }^{1,7}$, Thomas J. Cox ${ }^{4}$,

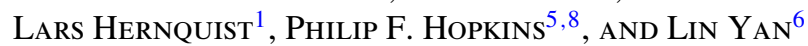 \\ ${ }^{1}$ Harvard-Smithsonian Center for Astrophysics, 60 Garden Street, Cambridge, MA 02138, USA; gsnyder@cfa.harvard.edu \\ ${ }^{2}$ Heidelberger Institut für Theoretische Studien, Schloss-Wolfsbrunnenweg 35, D-69118 Heidelberg, Germany \\ ${ }^{3}$ Department of Physics and Astronomy, Tufts University, 4 Colby Street, Medford, MA 02155, USA \\ ${ }^{4}$ Carnegie Observatories, 813 Santa Barbara Street, Pasadena, CA 91101, USA \\ ${ }^{5}$ Department of Astronomy, University of California at Berkeley, C-208 Hearst Field Annex, Berkeley, CA 94720, USA \\ ${ }^{6}$ Infrared Processing and Analysis Center, California Institute of Technology, Pasadena, CA 91125, USA \\ Received 2012 October 7; accepted 2013 April 5; published 2013 April 25
}

\begin{abstract}
We analyze the link between active galactic nuclei (AGNs) and mid-infrared flux using dust radiative transfer calculations of starbursts realized in hydrodynamical simulations. Focusing on the effects of galaxy dust, we evaluate diagnostics commonly used to disentangle AGN and star formation in ultraluminous infrared galaxies (ULIRGs). We examine these quantities as a function of time, viewing angle, dust model, AGN spectrum, and AGN strength in merger simulations representing two possible extremes of the ULIRG population: one is a typical gas-rich merger at $z \sim 0$, and the other is characteristic of extremely obscured starbursts at $z \sim 2-4$. This highly obscured burst begins star-formation-dominated with significant polycyclic aromatic hydrocarbon (PAH) emission, and ends with a $\sim 10^{9} \mathrm{yr}$ period of red near-IR colors. At coalescence, when the AGN is most luminous, dust obscures the near-infrared AGN signature, reduces the relative emission from PAHs, and enhances the $9.7 \mu \mathrm{m}$ absorption by silicate grains. Although generally consistent with previous interpretations, our results imply none of these indicators can unambiguously estimate the AGN luminosity fraction in all cases. Motivated by the simulations, we show that a combination of the extinction feature at $9.7 \mu \mathrm{m}$, the PAH strength, and a near-infrared slope can simultaneously constrain the AGN fraction and dust grain distribution for a wide range of obscuration. We find that this indicator, accessible to the James Webb Space Telescope, may estimate the AGN power as tightly as the hard X-ray flux alone, thereby providing a valuable future cross-check and constraint for large samples of distant ULIRGs.
\end{abstract}

Key words: dust, extinction - galaxies: interactions - galaxies: starburst - infrared: galaxies - quasars: general radiative transfer

Online-only material: color figures

\section{INTRODUCTION}

Understanding the link between supermassive black holes (SMBHs) and their host galaxies is essential for deciphering the formation and evolution of galaxies. Galaxy/SMBH co-evolution is expected given the observed correlations between SMBH mass and galaxy properties (e.g., Kormendy \& Richstone 1995; Magorrian et al. 1998; Ferrarese \& Merritt 2000; Tremaine et al. 2002; Gültekin et al. 2009) and theoretical arguments that SMBH growth is self-regulated by feedback (e.g., Silk \& Rees 1998; Springel et al. 2005; Hopkins et al. 2007a, 2007b).

A key prediction from this framework is that a galaxy experiencing rapid inflow of cold gas can evolve through various classes of starbursts and active galactic nuclei (AGNs), such as ultra-luminous infrared galaxies (ULIRGs) and quasars (QSOs), and that these phases are connected in an evolutionary sequence (e.g., Sanders et al. 1988). With such a model, both a starburst and a heavily obscured AGN can co-exist (Hopkins et al. 2006).

Testing this picture requires not only finding signatures of obscured AGN activity within starburst galaxies, but also interpreting them in the context of galaxy/SMBH co-evolution. Locally, the most extreme starbursts are the ULIRGs $\left(L_{\mathrm{IR}}>\right.$ $10^{12} L_{\odot}$; Sanders et al. 1988), which are almost exclusively the

\footnotetext{
7 Present Address: Space Exploration Technologies, 1 Rocket Rd, Hawthorne, CA 90250, USA.

8 Einstein Fellow.
}

result of a recent/ongoing major merger (Sanders \& Mirabel 1996), which triggers both starburst and obscured AGN activity. To evaluate SMBH growth and the role of feedback during this critical phase, it is necessary to robustly estimate the AGN power during all observed phases. A primary challenge to determine the fraction of flux attributable to the AGN is the reprocessing of its photons by the host galaxy's interstellar medium (ISM). In essence, most diagnostics focus on finding signatures that are directly associated with the AGN output: narrow-line region emission, X-rays, torus emission, or radio emission.

A popular approach to studying, in particular, dust-obscured AGNs utilizes the mid-infrared (mid-IR) regime, loosely defined as 3-30 $\mu \mathrm{m}$, where typical starbursts have a spectral energy distribution (SED) with a minimum in continuum emission, while continuum emission from dust surrounding AGNs rises toward longer wavelengths owing to hot dust emission, potentially from a torus (e.g., Pier \& Krolik 1992; Stern et al. 2005; Hönig et al. 2006; Nenkova et al. 2008).

This idea led to the construction of diagnostic diagrams separating starburst from AGN dominated sources, based on Infrared Space Observatory data of local IR-luminous sources (Lutz et al. 1996, 1998; Genzel et al. 1998; Rigopoulou et al. 1999; Laurent et al. 2000; Tran et al. 2001). The sensitive Infrared Spectrograph (IRS; Houck et al. 2004) aboard the Spitzer Space Telescope (Werner et al. 2004) enabled the expansion of this approach to larger samples covering a wider range of physical properties (e.g., Smith et al. 2007; Brandl 
et al. 2006; Schweitzer et al. 2006; Wu et al. 2006; Dale et al. 2006; Armus et al. 2006, 2007; Sturm et al. 2006; Spoon et al. 2007), and permitted mid-IR diagnostic work on objects at higher redshifts. In particular, mid-IR diagnostics have been applied to galaxies at $z \sim 1-3$ (e.g., Houck et al. 2005; Yan et al. 2007; Pope et al. 2008), the epoch of peak star-formation rate (SFR) density (e.g., Bouwens et al. 2007) and peak quasar number density (e.g., Richards et al. 2006b), which makes it a crucial period for evaluating the co-evolution of galaxies and SMBHs. It is also when LIRGs and ULIRGs (see Sanders \& Mirabel 1996 for a review) make a significant contribution to the global averaged luminosity density and to the cosmic infrared background (Le Floc'h et al. 2005; Dole et al. 2006; Caputi et al. 2007; Hopkins et al. 2010).

Other findings on high- $z$ IR-luminous galaxies suggest that high-redshift ULIRGs may be unlike local ULIRGs in that they may not all be late-stage mergers. For example, $z \sim 2$ sources tend to be more starburst-dominated than $z \sim 0$ sources of comparable mid-IR luminosity (Fadda et al. 2010). Differences in both the SEDs and morphologies of high redshift ULIRGs, as compared with local $(z \lesssim 0.1)$ sources, do suggest that they are not analogous (e.g., Sajina et al. 2012, hereafter S12). Furthermore, submillimeter galaxies, a subset of high-redshift ULIRGs, may be a mix of early-stage quiescently star-forming mergers, late-stage merger-induced starbursts, and isolated disk galaxies (Hayward et al. 2012, 2013). The typically greater gas fractions (Tacconi et al. 2010) imply that fueling both star-formation (SF) and black hole accretion is relatively easier, and therefore not necessarily analogous to what we see in local ULIRGs.

In both the local and high-redshift work, it is not clear to what extent common diagnostics miss the crucial evolutionary stage when the AGN is most deeply obscured. It is possible that we can only detect the presence of an AGN once enough unobscured lines of sight to the AGN exist. Moreover, while IR diagnostics generally follow the expected AGN/starburst fractions, the evolution of IR SED properties reflects a complex mix of these components which may be difficult to interpret for individual sources or samples (Veilleux et al. 2009, hereafter V09). Therefore it is desirable to search for signposts of AGN powering that may suffer minimally from these complexities.

To analyze this issue, numerical calculations have been used to capture the complex geometries and radiation sources relevant for disentangling AGN activity from SF. Originally restricted to simplified geometries (e.g., Witt et al. 1992), calculations of dust attenuation and reprocessing are now tenable for threedimensional dynamical simulations on galactic scales including starbursts and SMBH emission (e.g., Chakrabarti et al. 2007). Studies of hydrodynamical simulations with dust radiative transfer (RT) in postprocessing have found that AGN signatures, such as a power-law SED or warm IR colors in ULIRGs, are generally associated with SMBH activity (Younger et al. 2009) but may also be caused by intense starbursts (Narayanan et al. 2010a). Analyzing the predicted far-IR emission from such simulations, Hayward et al. $(2011,2012)$ found that the IR signatures of SF vary depending on whether the activity occurs in a quiescent or bursty mode, potentially complicating discriminators of AGN and SF activity. One challenge raised by this body of work is how to interface the modeled SMBH with the host galaxy when it is not feasible to model the central engine fully self-consistently, a problem similar to the one described by, e.g., Jonsson et al. (2010) for modeling young star clusters.
In this paper, we combine the high diagnostic power of midIR features, such as polycyclic aromatic hydrocarbons (PAH) emission and the $9.7 \mu \mathrm{m}$ silicate feature (e.g., Siebenmorgen et al. 2005; Levenson et al. 2007), with three-dimensional hydrodynamical galaxy merger simulations to better understand the existing Spitzer IRS data, and to prepare for future data from the James Webb Space Telescope (JWST). In this novel approach to AGN diagnostics, we calculate infrared SEDs from simulations using dust RT, including a simple model for AGN accretion and emission. We then use mid-IR diagnostics to gain insight regarding AGN activity when dust in the host galaxy may be important, and also to highlight areas in which such modeling techniques might be improved. In Section 2, we describe simulations of two representative starbursts meant to bracket the level of obscuration in ULIRGs, from which we compute and analyze the mid-IR SEDs as described in Section 3. In Section 4, we show how these features depend on AGN power, evolutionary stage, viewing direction, intrinsic AGN SED, and assumptions about the dust and ISM. In Section 5, we construct commonly used diagnostic diagrams and evaluate their ability to estimate the AGN luminosity fraction. We consider implications of this work for future AGN modeling and studies of the evolution of IR-luminous galaxies in Section 6, and we conclude in Section 7. We explore a toy model for our indicators in the Appendix.

\section{SIMULATIONS}

We combine high-resolution GADGET-2 (Springel 2005) 3-D N-body/smoothed-particle hydrodynamics (SPH) simulations of equal-mass galaxy mergers with the SUNRISE (Jonsson 2006; Jonsson et al. 2010) polychromatic Monte Carlo dust RT code to examine commonly-applied mid-infrared (mid-IR) AGN signatures. We focus on two representative mergers, a "highly obscured" hyper-LIRG analog, and a "less obscured" marginal ULIRG example. The highly obscured simulation analyzed here is the same one focused on in Hayward et al. (2011).

\subsection{Hydrodynamical Simulations}

GADGET-2 is a TreeSPH (Hernquist \& Katz 1989) code that conserves both energy and entropy (Springel \& Hernquist 2002). The simulations include radiative heating and cooling as in Katz et al. (1996), which assumes ionization equilibrium with an ultraviolet background (Faucher-Giguère et al. 2009) and no metal line cooling. SF is modeled to reproduce the global Kennicutt-Schmidt law (Kennicutt 1998) via the volumetric relation $\rho_{\text {SFR }} \propto \rho_{\text {gas }}^{1.5}$ with a minimum density threshold $n \sim$ $0.1 \mathrm{~cm}^{-3}$. The $\mathrm{SF}$ law employed should be considered an empirically and physically motivated prescription to summarize physics we do not resolve. We do not track the formation of molecular gas or resolve individual molecular clouds, and this limitation is a key uncertainty in our RT step (see Section 2.3).

The structure of the ISM is modeled via a two-phase sub-resolution model in which cold, star-forming clouds are embedded in a diffuse, hot medium (Springel \& Hernquist 2003) pressurized by supernova feedback that heats the diffuse ISM and evaporates the cold clouds (Cox et al. 2006b). All supernova energy is assumed to thermally heat the hot ambient medium, and no kinetic stellar wind kicks are used. Metal enrichment is calculated by assuming each gas particle behaves as a closed box with a yield $y=0.02$. SMBH particles accrete via Eddingtonlimited Bondi-Hoyle accretion and deposit $5 \%$ of their luminosity to the nearby ISM as thermal energy (Springel et al. 2005; 
Table 1

Properties of Merger Simulations

\begin{tabular}{|c|c|c|c|c|c|c|c|}
\hline Name & $M_{\text {halo }} / M_{\odot}$ & $M_{\text {disk }} / M_{\odot}$ & $f_{\text {gas,init }}$ & $M_{\mathrm{BH}} / M_{\odot}(t=0)$ & $M_{\mathrm{BH}} / M_{\odot}\left(t=t_{4}\right)$ & Halo $N_{\text {part }}{ }^{\mathrm{a}}$ & Disk $N_{\text {part }}$ \\
\hline Highly obscured $^{\text {b }}$ & $9 \times 10^{12}$ & $4 \times 10^{11}$ & 0.6 & $1.4 \times 10^{5}$ & $9.6 \times 10^{8}$ & $6 \times 10^{4}$ & $8 \times 10^{4}$ \\
\hline Less obscured ${ }^{c}$ & $2.6 \times 10^{12}$ & $1.1 \times 10^{11}$ & 0.4 & $1.4 \times 10^{5}$ & $8.7 \times 10^{7}$ & $1.2 \times 10^{5}$ & $8 \times 10^{4}$ \\
\hline
\end{tabular}

Notes.

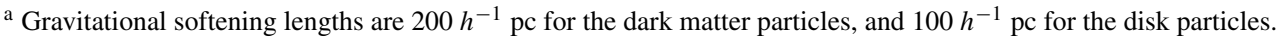

${ }^{b}$ Galaxy properties scaled to $z \sim 3$ following Robertson et al. (2006).

${ }^{\mathrm{c}}$ Galaxy properties assumed typical for $z \sim 0$.

Di Matteo et al. 2005). The luminosity is computed from the accretion rate assuming $10 \%$ radiative efficiency, $L_{\text {bol }}=0.1 \dot{M} c^{2}$.

In this paper, we focus on two merger simulations, summarized in Table 1, that represent starbursts with different characteristics. Our "highly obscured" simulation is a very massive, gas-rich merger meant to mimic very luminous starbursts at $z \sim 2-4$, while the "less obscured" simulation is more representative of typical gas-rich mergers at $z \sim 0$. In each case, we use two identical progenitor galaxies. The progenitors of the highly obscured merger are composed of an exponential disk with baryonic mass $4 \times 10^{11} M_{\odot}(60 \%$ of which is gas) and a dark matter halo of mass $9 \times 10^{12} M_{\odot}$ described by a Hernquist (1990) profile. The galaxy properties are scaled to $z \sim 3$ following Robertson et al. (2006). The orbital parameters are identical to those of the " $e$ " orbit of Cox et al. (2006a). In contrast, the progenitors of the weakly obscured example have lower mass $\left(M_{*} \sim 10^{11} M_{\odot}\right)$, are less gas-rich $(40 \%)$ than the highly obscured example, and are scaled to $z \sim 0$. Each progenitor galaxy is seeded with one SMBH particle.

In this work, we focus on signatures of AGN activity that normalize out the galaxy's mass or total luminosity, and therefore the salient differences between these two cases are the relative extents to which the merger triggers SF, AGN activity, and obscuration. A disadvantage with this approach is that we are restricted in the range of ULIRG scenarios we can probe, owing in part to limited computational resources. In this paper, we have chosen to explore two merger simulations that potentially reflect the extremes of IR-luminous galaxies, and we discuss further consequences of this limitation in Section 2.4.

\subsection{Radiative Transfer}

We use SUNRISE $^{9}$ Version 3 in post-processing to calculate the SED observed from seven cameras distributed isotropically in solid angle every 10 Myr. For a full description of SuNRISE see Jonsson (2006) and Jonsson et al. (2010), and for a summary of components essential for this work and a description of other specific choices, see Hayward et al. (2011). We describe specific choices for some of the RT parameters in Section 2.3.

\subsubsection{Sources}

SUNRISE calculates the emission from the stars and AGNs in the GADGET-2 simulations and the attenuation and re-emission from dust. STARBURST99 (Leitherer et al. 1999) SEDs are assigned to star particles according to their ages and metallicities, and SMBH particles emit the luminosity-dependent templates of Hopkins et al. (2007c) by assuming the formula above for $L_{\text {bol }}$. At mid-IR wavelengths, this template emits the mean SED of Richards et al. (2006a). In Section 4.4, we vary the AGN

\footnotetext{
9 See http://code.google.com/p/sunrise/ for the project source code and documentation.
}

source SED, applying two clumpy torus models by Nenkova et al. (2008) that span their modeled properties. Our templates follow typical bright AGNs in that only $\lesssim 10 \%$ of their flux is emitted at X-ray wavelengths

Recently formed star particles $\left(t<10^{6} \mathrm{yr}\right)$ are assigned the MAPPINGSIII H II region sub-grid SED models of Groves et al. (2008). These models include a dusty photodissociation region (PDR) with a tunable covering fraction $\left(f_{\mathrm{PDR}}\right)$. With a non-zero $f_{\mathrm{PDR}}$, the dust re-emission from such regions is included in the emission template. In these models, a fraction of the carbon grains are assumed to be PAH molecules whose absorption is calculated from the cross sections by Draine \& Li (2001). Their emission is modeled to follow a fixed template of Lorentzian profiles designed to match Spitzer IRS observations of PAH emission (Dopita et al. 2005; Groves et al. 2008). We use $f_{\mathrm{PDR}}=0$ for the "highly obscured" simulation owing to concerns about the applicability of these templates to the extreme ISM densities and pressures found in this merger; see Section 2.2.1 of Hayward et al. (2011) for a full discussion of these issues.

\subsubsection{ISM Structure, Metals, and Dust}

To initialize the galaxy dust RT calculation, SUNRISE projects the GADGET-2 gas-phase metal density onto a three-dimensional Cartesian adaptive grid. To construct this grid, the code bounds the simulation volume with a $(200 \mathrm{kpc})^{3}$ cube and divides this initial cube into 125 equally sized subregions for the first level of refinement. Subsequent refinement is based on an estimate for the dust optical depth in each cell from the GADGET metal density. In this work, nine further refinement steps were undertaken, in which cells targeted for refinement are split into eight sectors by dividing each spatial dimension in half. This leads to a minimum cell size of $(200 \mathrm{kpc} / 5) / 2^{9}=80 \mathrm{pc}=$ $55 \mathrm{~h}^{-1} \mathrm{pc}$, roughly half of the softening length $\left(100 h^{-1} \mathrm{pc}\right)$ used for the hydrodynamical simulations, or $\sim 1 / 4$ of the SPH resolution. For the fixed resolution of the hydrodynamical simulations, this level of refinement ensures that the SEDs are converged to within 10\% (Hayward et al. 2011), indicating that our RT grid settings are satisfactory for the simulations we use here.

The galaxy stars and gas were assumed to have an initial mass fraction in metals of $Z=0.01$. During the mergers presented here, the mean gas-phase mass fraction of metals increases to $Z \sim 0.018$ just after the merger coalescence. Here we assume $40 \%$ of these metals are in dust (Dwek 1998), giving a dustto-gas ratio of $\sim 1 \%$ for solar metallicity gas. We consider the Milky Way $R_{V}=3.1$ (MW) and SMC bar (SMC) dust models of Weingartner \& Draine (2001), updated by Draine \& Li (2007). These models are comprised of populations of carbonaceous and silicate dust grains inferred from observations along lines of sight to stars in the two regions. The Small Magellanic Cloud 
(SMC) dust grain model we apply differs from the MW model in part by having a factor of $\sim 10$ fewer small carbonaceous grains (including, e.g., the PAHs), leading to a much weaker $2175 \AA$ absorption feature. In active regions, small grains are expected to be depleted by a number of processes (e.g., Laor \& Draine 1993) on short timescales, and it has been found that galactic dust appears to vary continuously between properties that are MW-like and those that are SMC-like owing to the influence of SF activity (e.g., Gordon et al. 2003 and references therein). Draine et al. (2007) found that typical nearby spirals tend to have dust properties similar to those that give rise to the MW dust model, while bright AGN are believed to be depleted of small grains (Laor \& Draine 1993). Hopkins et al. (2004) found that an SMC-like dust model better matches reddening of the optical colors in quasars. Observations of nearby starburst galaxies were found to lack a strong $2175 \AA$ extinction feature, also resembling SMC dust (Gordon et al. 1997; Gordon \& Clayton 1998). Likewise, Vijh et al. (2003) found that the SEDs of $z \sim 3$ starbursts (e.g., Lyman break galaxies) indicate SMC-like dust.

Therefore, the observed dust properties are correlated with the evolutionary stage of the galaxy, as SF and AGN activity affect the creation and destruction of grains. We limit ourselves to specifying the dust model by hand for these MW and SMC models, but in principle an evolving model could be developed and tested against observations using a suite of simulations like those we analyze here.

\subsubsection{Radiative Transfer}

With the dust distribution and grain models set, SUNRISE then performs Monte Carlo RT through the galaxy dust by emitting photon packets from the sources and drawing interaction optical depths from the appropriate probability distribution as the packets traverse the ISM. For each grid cell, the temperature of each dust species (with the exception of PAHs) is calculated assuming the dust is in thermal equilibrium, and the dust re-emits the absorbed energy as a modified blackbody. PAH molecules are treated using a method similar to the MAPPINGSIII models. A fixed fraction, $f_{t}$, of the carbon grains with size $a<100 \AA$ are assumed to emit radiation thermally. The remaining fraction, $1-f_{t}$, are assumed to be PAH molecules that emit the template spectrum from Groves et al. (2008). $f_{t}$ is a free parameter that we set to $f_{t}=0.5$ following Jonsson et al. (2010), roughly matching the $8 \mu \mathrm{m}-24 \mu \mathrm{m}$ flux ratios from the Spitzer Infrared Nearby Galaxies Survey (Dale et al. 2007). In high-density regions, the dust can be opaque to its own emission, so the contribution of the dust emission to dust heating must be considered. SUNRISE computes this self-consistently by iteratively performing the transfer of the dust emission and the temperature calculation using a reference field technique.

SUNRISE calculates energy absorption by dust from radiation at wavelengths $912 \AA<\lambda<1000 \mu \mathrm{m}$, neglecting dust heating by radiation at energies above the Lyman limit. This may neglect some amount of energy absorbed from X-ray radiation $(h v>0.5 \mathrm{keV})$ by atoms in dust grains. However, since our AGN template emits $\lesssim 10 \%$ of its energy at these wavelengths, and the dust may not thermalize effectively under radiation at these wavelengths, we expect that the contribution to thermal dust emission we ignore from this regime can comprise only a few percent of the bolometric luminosity (Laor \& Draine 1993). Additionally, SunRISE neglects the effects of thermal fluctuations in stochastically heated galaxy dust grains.

Our focus herein is the mid-IR portion of the rest-frame SEDs, $1 \mu \mathrm{m}<\lambda<30 \mu \mathrm{m}$, a regime in which dust grains heated to a wide range of temperatures $(T \sim 100-1500 \mathrm{~K})$ emit thermal radiation. Arbitrary dust temperature distributions are possible during a gas-rich galaxy merger, owing to the complex and chaotically-evolving multi-phase structure of the ISM induced during a global starburst and feedback-regulated SF and SMBH growth. Thus the accurate dust heating calculations employed by SUNRISE, including treatments of multiple dust species, are essential for understanding this key phase of galaxy evolution.

Since we will make extensive use of the $9.7 \mu \mathrm{m}$ silicate absorption feature, we stress that the depth of the feature is determined self-consistently via the RT. The strength of the feature depends on the amount of dust and the geometry of sources (stars and AGNs) and dust. For reasonable galaxy geometries we expect the AGNs to be more obscured than the bulk of the stars; this differential extinction is inherently ignored when one assumes a foreground screen dust geometry, so our simulations provide an excellent way to test the uncertainties caused by this assumption.

The results of the SUNRISE calculation are spatially resolved, multi-wavelength SEDs observed from seven directions. The success of this approach at modeling diverse galaxy populations-both local (e.g., Younger et al. 2009; Bush et al. 2010; Snyder et al. 2011) and high-redshift (e.g., Wuyts et al. 2010; Narayanan et al. 2010a, 2010b; Hayward et al. 2012, 2013) - lends credibility to its application here.

\subsection{Alternate Radiative Transfer Models}

In order to gain additional physical insight, we consider different sets of assumptions for the RT stage, including two sub-resolution models for the dust distribution on scales below that resolved by the GADGET-2 simulation. For our highly obscured simulation, we will focus on the ISM treatment referred to in Hayward et al. (2011) as "multi-phase off." This choice assumes that the dust mass contained in both phases of the Springel \& Hernquist (2003) ISM model is distributed uniformly across each resolution element, which is likely appropriate for gas-rich mergers in which the central regions are composed of dense, almost exclusively molecular gas. Throughout this work, we refer to this model as our "default ISM" treatment.

Alternatively, we can assume that gas in the cold, dense clouds of the Springel \& Hernquist (2003) model has a negligible volume filling factor. This choice retains the dust in the "hot" phase, which is assumed to have a volume filling factor equal to unity. Specifically, the dust mass that corresponds to this diffuse gas phase of the Springel \& Hernquist (2003) model is distributed uniformly across each resolution element, while the dust mass occupying the dense, cold clouds is ignored. In this case, we neglect attenuation and emission from both the cold clouds in which stars are formed and from other clouds photons encounter along the line-of-sight. This assumption thus gives a lower limit on the amount of attenuation. We refer to this assumption as our "alternate ISM" treatment, and apply it to both our highly and weakly obscured simulations.

The fraction of gas in the cold phase, and therefore the amount of dust ignored by the alternate ISM model, varies with time and position during our simulations. For the highly obscured merger, the amount of mass in the cold phase is $\sim 38 \%(\sim 27 \%)$ at the peak of the starburst (AGN). For the less obscured merger, this fraction is $\sim 28 \%$ at the peak starburst and $\sim 15 \%$ at peak AGN power. Gas which is at higher densities contains a larger fraction in the cold phase, and so during merger coalescence gas in the central few kpc achieves a cold phase fraction of $\sim 90 \%$. Thus the alternate ISM assumption, discarding this fraction of dust in 
the cold clouds, more strongly affects the central regions than the galaxy as a whole. Our RT calculation implicitly assumes that this dust does not absorb any energy, and therefore it is not included in the IR emission calculation. This dust emission would primarily affect far-IR wavelengths, and so it should not affect the analysis presented here.

The two treatments of subresolution dust structure we employ should be considered two plausible and physically-motivated, yet uncertain, ISM models that encapsulate unresolved processes. With current simulations, it is only possible to parameterize our ignorance in this way. However, any model used to interpret ULIRG SEDs is limited by this uncertainty. These simulations are thus particularly useful for their ability to quantify the uncertainty caused by dust clumpiness.

For both mergers, we perform the RT calculations after multiplying the SMBH luminosity at all times by zero $(\mathrm{AGN} \times 0)$, one $(\mathrm{AGN} \times 1)$, and ten $(\mathrm{AGN} \times 10)$, allowing us to manually adjust the AGN contribution to the SEDs. Note that the effect of AGN feedback, the thermal heating of the ISM surrounding the SMBH particles, is kept fixed at the fiducial level of Section 2.1. The AGN contribution to the mid-IR SED arises from separate self-consistent RT calculations with each of our assigned AGN luminosities, dust grain models, and ISM assumptions. This enables us to cleanly test how a given indicator depends on AGN luminosity for fixed geometry and galaxy ISM conditions. At each of these AGN strengths, we apply both ISM assumptions to the highly obscured merger and the "alternate ISM" treatment for the less obscured case. For these three sets of three simulations, we perform the RT using both the MW and SMC bar dust models.

\subsection{Galaxy Models}

Although the "high obscuration" and "low obscuration" simulations are meant to mimic gas rich starbursts in massive major mergers at $z \sim 3$ and $z \sim 0$, respectively (Table 1), these cases do not necessarily accurately reflect the real ULIRG population at a given redshift. The "high obscuration" example is, by choice, a particularly massive and gas-rich merger, and thus becomes a luminous hyper-LIRG that reaches a peak $\log L_{\mathrm{IR}} \sim 13$ briefly, having $\log L_{\mathrm{IR}}>12$ for $\sim 1$ Gyr. This merger is consistent with extreme starbursts such as sub-millimeter galaxies (SMGs) and hot-dust ULIRGs at $z \sim$ 2-4 (Hickox et al. 2012; Hayward et al. 2012, 2013), and therefore reflects a typically observed source at these redshifts (note: this does not mean it is a typical galaxy). By contrast, the "weakly obscured" example is marginally a ULIRG, briefly reaching $L_{\mathrm{IR}} \sim 10^{12} L_{\odot}$ at merger coalescence under our default assumptions, consistent with the idea that local ULIRGs are almost exclusively ongoing mergers (Sanders \& Mirabel 1996). Note that the initial gas fractions assigned in our progenitors, $60 \%$ and $40 \%$, are high compared to comparable star-forming galaxies at these two epochs - this is to account for our neglecting cosmological accretion and gas recycling, and a fairer comparison is to the gas fractions at the time of merger, which are $\sim 30 \%$ and $\sim 20 \%$.

Of course, real IR-luminous galaxy samples at a given redshift will draw from a wide range of scenarios. Therefore, we cannot hope to fully represent the ULIRG population in the present study. However, we will focus primarily on the goal of separating AGNs from SF activity in a small set of experiments defined by our two mergers with the RT variants described in Section 2.3, whose properties may more broadly span plausible situations. As an example, at $z \sim 3$, there are some mergers with the same stellar mass as our highly obscured simulation but a lower gas fraction. In the Eddington-limited phase, such mergers may have higher AGN-to-SF fractions than our fiducial highly obscured calculation, so the AGN $\times 10$ model may more accurately represent such cases. Similarly, our simulations at $z \sim 0$ may not be a close match to the conditions in all local ULIRGs. Moreover, while the final mass of our accreting SMBHs depends primarily on the fraction of feedback energy coupled to the ISM (e.g., Springel et al. 2005), the growth history of a SMBH, and hence its luminosity at a given time, can depend sensitively on the adopted sub-resolution model.

Given these uncertainties, the goal of our experiments-in particular the $\mathrm{AGN} \times 0, \mathrm{AGN} \times 1$, and $\mathrm{AGN} \times 10$ models-is to cover our bases and controllably boost the luminosity so that we span a wide enough range in the ratio of AGN-toSF activity, and not to comprehensively predict the behavior of observed ULIRGs. In the future, a more realistic treatment of the population may be possible as large suites of highresolution simulations become more common. However, many uncertainties remain not only in the physical models of the ISM and AGN accretion on sub-resolution scales, but also in how to interface emission and dust attenuation from the central engine with the host galaxy in the RT stage (e.g., Jonsson et al. 2010). Therefore, we begin with an exploration of these few numerical experiments in a first attempt to explore the applicability of this modeling technique.

In Figure 1, we summarize the predicted SEDs of our mergers. We focus on four times during each merger spanning 500 Myr, and present the total mid-IR SEDs emerging in a particular direction. In the left SED columns, we show the results of our fiducial ISM assumption, but artificially vary the total luminosity of the two (and eventually one) SMBH particles. In the right SED columns, we explore our assumptions about the ISM and dust, a study that we analyze in more detail below in Section 4.2 (see also Section 2.3).

Alongside the SEDs, we show high-resolution false-color composite images corresponding to the same viewing direction as the highly obscured SEDs. The left images present the system in the $U, V$, and $J$ bands (Johnson \& Morgan 1953), and the second in the 3.6, 4.5, and $8 \mu \mathrm{m}$ channels of the Spitzer Infrared Array Camera (IRAC; Fazio et al. 2004). These images provide some insight into how these systems might be classified in terms of a merger-stage diagnostic - if this system is observed at high redshift, then the only time it is obviously a merger is at $t \lesssim t_{1}$. This precedes the SMBH's peak luminosity and therefore the times at which it makes an obvious contribution to the mid-IR SED, a period spanning $\sim 1$ Gyr.

\subsection{X-Ray Calculations}

We compute X-ray fluxes from SMBH particles and subsequent attenuation by the ISM following the approach described by Hopkins et al. (2005, 2006). This method uses the intrinsic quasar SED (Section 2.2 and Hopkins et al. 2007c), and calculates its extinction at X-ray wavelengths by applying the photoelectric absorption cross sections of Morrison \& McCammon (1983), as well as Compton scattering cross sections, each scaled by metallicity.

A key difference between Hopkins et al. (2005) and the present work is that their assumptions correspond to the "alternate ISM" treatment we described in Section 2.3, where the cold clumps in the ISM, and hence often a significant fraction of the dust mass, are assumed to have a small volume filling factor. This may be true under many conditions, but may not be 

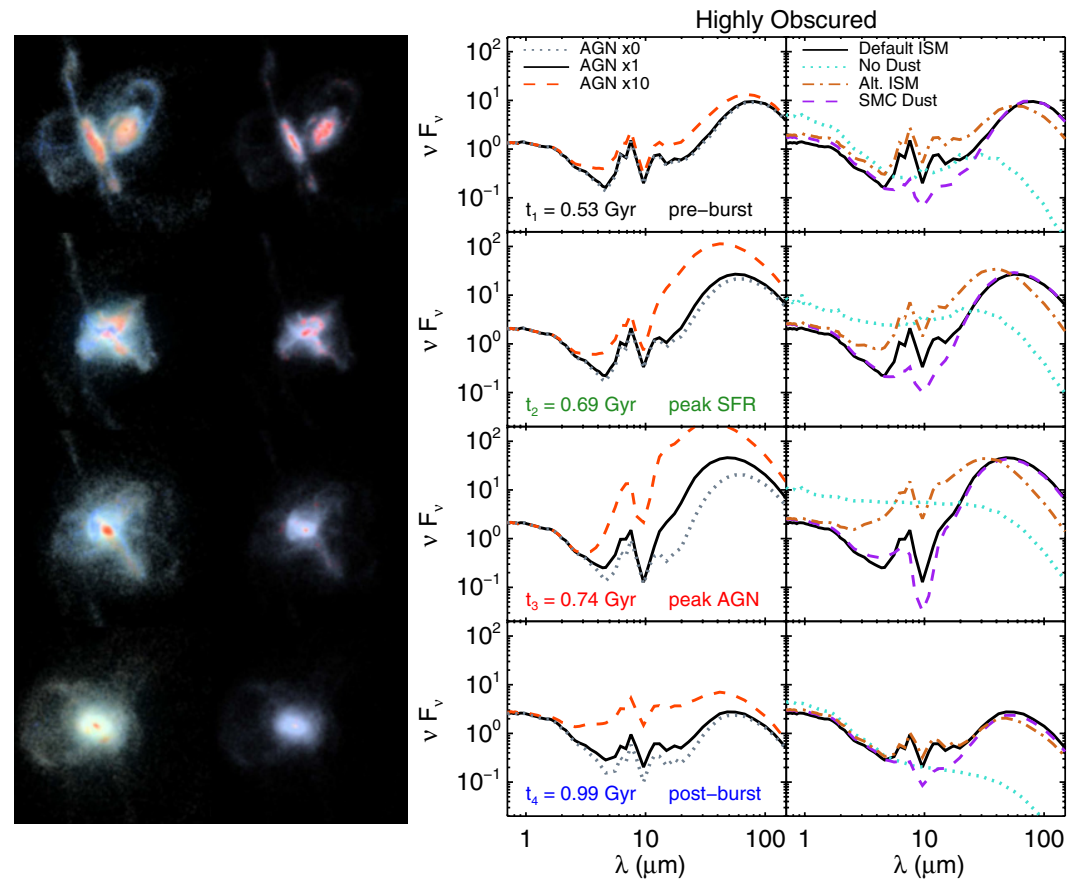

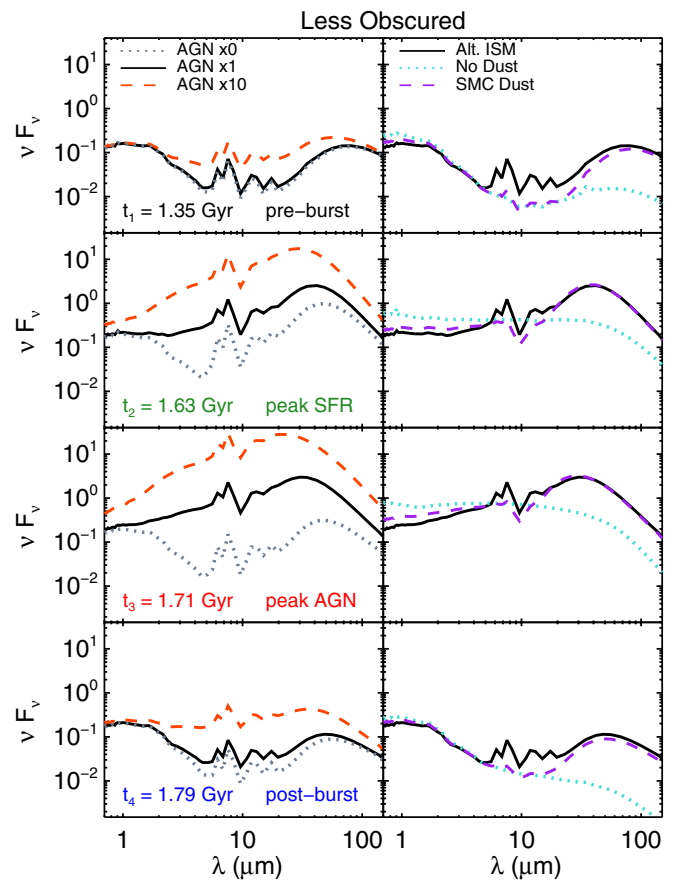

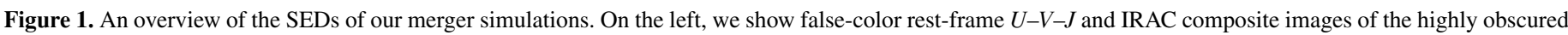

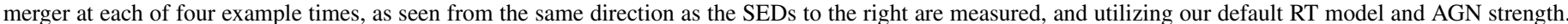

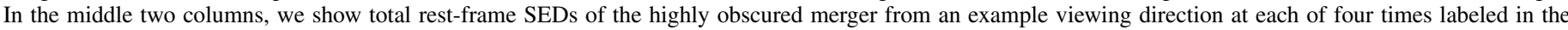

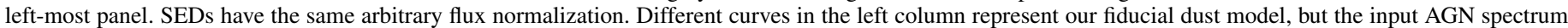

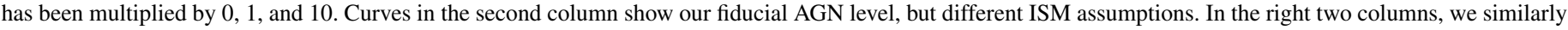
demonstrate the SEDs of the less obscured merger.

(A color version of this figure is available in the online journal.)

applicable to extremely gas-rich ULIRGs with abundant supplies of molecular gas. Therefore, we choose to use the same assumptions for X-ray attenuation that we use for the IR calculations described in Section 2.3. For our highly obscured merger simulation we calculate the column densities that attenuate the X-ray flux both ways: by discarding the cold phase mass ("alternate ISM"), and by keeping the cold phase mass ("default ISM"). For our less obscured merger simulation, we use only the "alternate ISM" model, discarding the cold phase mass.

\subsection{AGN Fraction}

For this paper, we focus on the AGN fraction, which we define as the ratio of SMBH luminosity to total luminosity across the wavelength range used by SuNRISE: $0.09-10^{3} \mu \mathrm{m}$. We denote this quantity $L_{\text {agn }} / L_{\text {bol }}$. Our definition uses the intrinsic AGN power regardless of how it appears in the final observed SED (i.e., it is insensitive to the amount of attenuation), but it does not include AGN emission at X-ray wavelengths. This quantity, $L_{\text {agn }} / L_{\text {bol }}$, is not normally available for a given observed sample. Here, the SMBH accretion rate and $L_{\text {agn }} / L_{\text {bol }}$ are available directly from the GADGET and SunRISE calculations. Thus we can directly evaluate the effectiveness of observed indicators, defined in Section 3, at estimating the AGN contribution utilizing self-consistent calculations of the galaxy's stars, dust, and SMBH.

\section{MID-INFRARED SPECTRAL DIAGNOSTICS}

\subsection{From Simulations}

We compute mid-infrared diagnostics based on the rest-frame spatially integrated SUNRISE SEDs, which we store as specific luminosities, i.e., the energy through the camera per unit time and per unit frequency (or wavelength) interval: $L_{v}$ or $L_{\lambda}$. For simplicity and for analogy with observable quantities, we denote these as $f_{\lambda} \equiv L_{v}(\lambda)$, where $\lambda$ is in microns. Owing to computational constraints, these SEDs have very low spectral resolution $(\lambda / \Delta \lambda \sim 10)$, so we limit consideration to approximations of several observationally-motivated estimators. We focus on two spectral dust features, $r_{9.7}, r_{7.7}$, defined by

$$
\begin{gathered}
r_{9.7}=\frac{f_{9.7}}{c_{9.7}} \\
r_{7.7}=\frac{f_{7.7}}{c_{7.7}},
\end{gathered}
$$

where $c_{\lambda}$ is the continuum level estimated by interpolating linearly between $f_{5.7}$ and $f_{15}$. The remaining indicators are ratios of $f_{\lambda}$ at $1.6,5.7,15$, and $30 \mu \mathrm{m}$. These points were chosen to match common mid-IR colors, in which a point is often chosen just below $6 \mu \mathrm{m}$ to avoid the $6.2 \mu \mathrm{m}$ PAH and $6 \mu \mathrm{m}$ water-ice features. Unless otherwise specified, we present all SEDs and their derived quantities in the source's rest frame.

In Figure 2, we present an example rest-frame SED in the mid-IR and highlight the spectral features that we will consider here. In addition to the final SED, we plot the intrinsic SMBH SED that we assume, which exhibits a generally flat shape with an "IR bump." If this source were observed unobscured, or obscured only by an optically thin (at several $\mu \mathrm{m}$ ) dust column, then this would resemble a "power-law-like" AGN source with red near- and mid-IR colors. We see this situation in the $t_{2}$ and $t_{3}$ panels for the less obscured merger (right side) of Figure 1: the $f_{4.5} / f_{1.6}$ slopes depend strongly on the AGN strength, and a comparison of the "no dust" and "alternate ISM" cases indicates that dust attenuation is insignificant at wavelengths longer than 


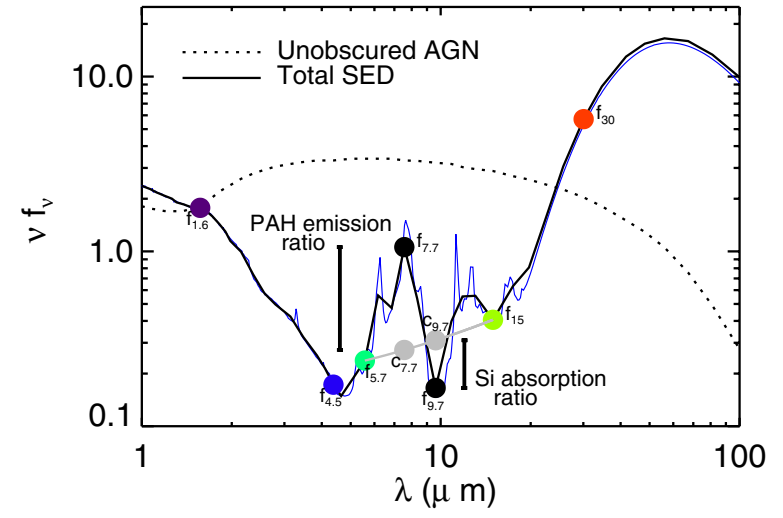

Figure 2. In the solid black line, we show an example SED output from SUNRISE in the source's rest frame. For all subsequent analysis we use this low-resolution version, but here we plot in blue a higher-resolution calculation that demonstrates the validity of the continuum estimation that we show in gray (see Section 3). In the dashed black curve, we show the intrinsic AGN spectrum used by SUNRISE as a light source from the SMBH particles. This SED occurs between the peak of the starburst and the peak of the AGN's bolometric contribution to the SED (between times $t_{2}$ and $t_{3}$ shown in Figure 1). Note that during this time the intrinsic AGN emission (attributed to the torus) at $2 \mu \mathrm{m}<\lambda<10 \mu \mathrm{m}$ is completely absorbed and reprocessed into the far-infrared.

(A color version of this figure is available in the online journal.)

a few microns. Furthermore, a near-IR slope (such as $f_{4.5} / f_{1.6}$ ) has been used to select AGN in wide-field IR surveys (see, e.g., Stern et al. 2005, 2012). Thus we will keep this feature in mind while analyzing the mid-IR properties of more highly obscured sources.

By contrast, the starburst SED falls as lambda increases at $1 \mu \mathrm{m}<\lambda<10 \mu \mathrm{m}$, but also exhibits distinctive features from dust grains identified as PAHs superimposed at 3.3, 6.2, 7.7, 8.6, 11.3, 12.6, and $17 \mu \mathrm{m}$. For example, SEDs characteristic of starbursts are seen in the first row of SEDs in Figure 1 (i.e., the black curves at time $t_{1}$ ).

\subsection{From Observations}

Observationally, weaker PAH and stronger continuum (i.e., lower PAH equivalent width-EW) effectively indicate a higher relative contribution from the AGN in dusty galaxies, especially ULIRGs (e.g., Laurent et al. 2000; Sturm et al. 2000; Tran et al. 2001). This diagnostic is supported by mid-IR fine structure line diagnostics implying an AGN-like radiation field in sources of lower PAH EW (Genzel et al. 1998; Armus et al. 2007).

More recently, such mid-IR diagnostic studies discovered sources that have a small PAH EW but a deep $9.7 \mu \mathrm{m}$ silicate absorption feature (e.g., Houck et al. 2005), which seems to require a deeply embedded, centrally concentrated source (Levenson et al. 2006). These sources are believed to represent Compton-thick AGNs (Bauer et al. 2010; Georgantopoulos et al. 2011), although there is at least one known star forming dwarf without an AGN whose mid-IR spectrum fits the above criteria (Roussel et al. 2003).

In Section 5, we will compare our predictions with data for local and high- $z$ starbursts with available low-resolution Spitzer IRS spectra. Specifically, we use the ULIRG sample of V09 and a sample of $19224 \mu \mathrm{m}$-selected $z \sim 0.3-3$ starbursts and obscured quasars. The redshifts and IRS spectra of the $24 \mu \mathrm{m}$-bright sample are presented in Yan et al. (2007) and Dasyra et al. (2009), while the full IR SEDs are compiled in $\mathrm{S} 12$.
Using the IRS spectra for both ULIRG samples, we estimate $r_{7.7}, r_{9.7}, f_{6}, f_{15}$, and $f_{30}$ in the same manner as for the simulated SEDs. However, the observed spectra have a more limited spectral coverage. The observed coverage of the IRS spectra varies between 5.2 and $38 \mu \mathrm{m}$, or $14-38 \mu \mathrm{m}$ if only the Long-Low modules are used, as is the case for most of the higher- $z$ sample. This means that for the local ULIRG sample, the required rest-frame $6-30 \mu \mathrm{m}$ is covered by the available IRS spectra, while for the $24 \mu \mathrm{m}$-bright sample, the $15 \mu \mathrm{m}$ and $30 \mu \mathrm{m}$ continuum points are outside the IRS coverage above $z \sim 1.5$. Below $z \sim 1.5$, the $6 \mu \mathrm{m}$ continuum point is often outside the IRS coverage (depending on whether or not the ShortLow module is available), and even the $7.7 \mu \mathrm{m}$ point can be outside the IRS coverage below $z \sim 0.8$. We compensate using the empirical SED model fits from S12, which necessarily introduces additional systematic uncertainty. These extrapolations are constrained below or above the IRS spectral coverage with IRAC 3.6, 4.5, 5.8, and $8 \mu \mathrm{m}$, and MIPS $70 \mu \mathrm{m}$ photometric points, ensuring that the mid-IR colors obtained are reasonably accurate.

We choose to exclude the $z \lesssim 0.9$ sources from this sample in order to avoid all cases where the $7.7 \mu \mathrm{m}$ PAH feature is not covered by the IRS spectra. This step also removes all sources whose overall IR luminosities $\left(L_{3-1000}\right)$ place them in the LIRG rather than ULIRG category. We also exclude the $z>2.5$ sources, for which the $9.7 \mu \mathrm{m}$ silicate absorption feature is too poorly defined, being only partially covered by the IRS spectra. This leaves a total of 118 sources. We explore determining the above quantities with and without interpolating the IRS spectra onto the much lower resolution simulated SED wavelength array and we find no significant difference in the overall results.

Lastly, in four cases, the silicate feature is saturated, making the measured $r_{9.7}$ value a lower limit. The saturation flag is triggered when the mean of the IRS spectra between 9.0 and $10.4 \mu \mathrm{m}$ is less than or equal to the standard deviation in the same region.

In Section 6, we discuss the rest-frame near-IR as another means of diagnosing AGN power. For observational comparisons in this regime, we use Spitzer IRAC photometry for the high-redshift sample, and for the low-redshift ULIRGs, we interpolate from Two Micron All Sky Survey (2MASS) $H$ - and $K$-band photometry (using total magnitudes). The unknown aperture corrections add some uncertainty here; however, ULIRGs are typically compact enough in the near-IR relative to the $\sim 2.5$ arcsec 2MASS beam that aperture effects should be small (Surace \& Sanders 1999; Surace et al. 2000). For several sources, we use 2.5-5 $\mu \mathrm{m}$ spectra (Imanishi et al. 2008; Sajina et al. 2009) from $A K A R I$ for more accurate near-IR fluxes.

\section{SIMULATION RESULTS}

\subsection{Time Evolution}

In Figure 3, we explore how the mid-IR diagnostics vary with time for our two fiducial simulations: "highly obscured" and "less obscured." We show spectral diagnostics along with quantities of interest such as the overall IR luminosity, the $\mathrm{SFR}$, and the AGN fraction, $L_{\mathrm{agn}} / L_{\mathrm{bol}}$. We assume our default ISM model and default AGN torus model, but we show the effect of varying these choices in Section 4.2 and Section 4.4, respectively. All curves shown are the median over the viewing angle. The scatter introduced by the uncertainty in the viewing angle is addressed in Section 4.3. 


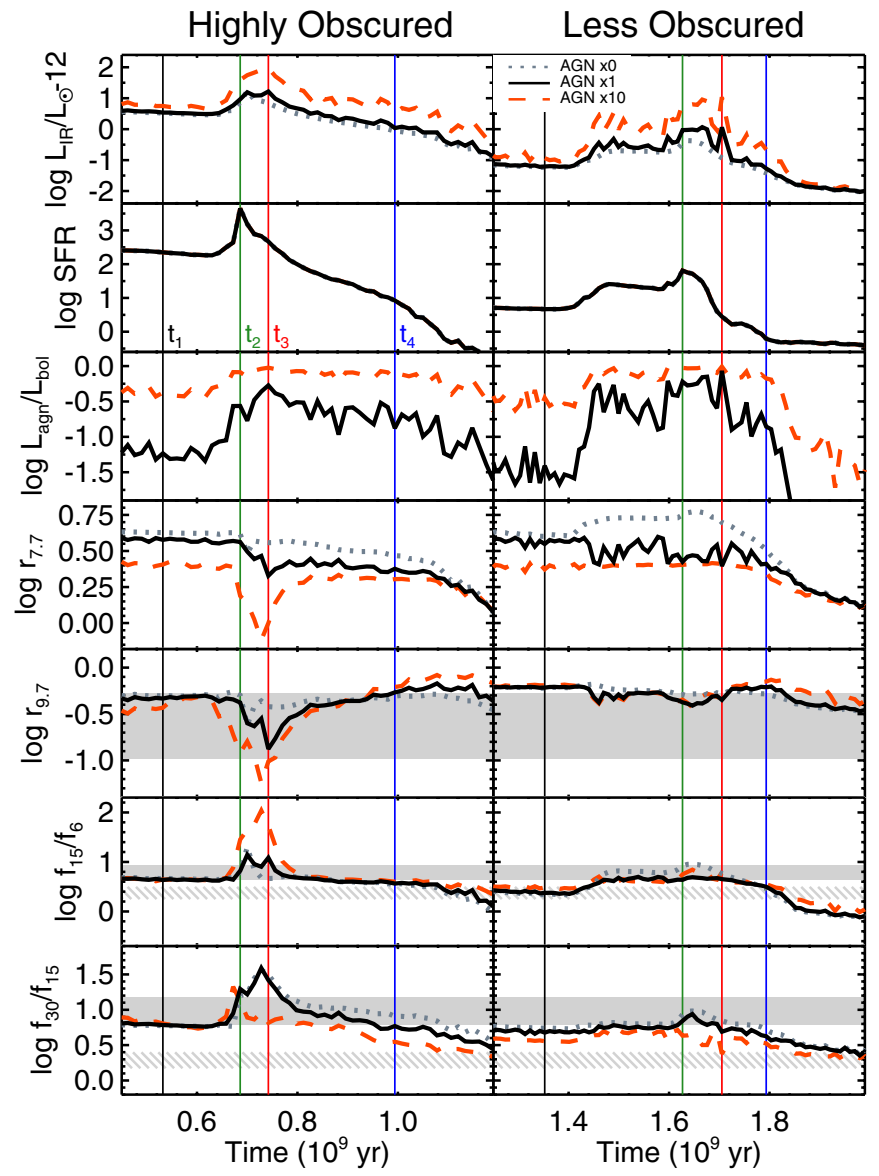

Figure 3. We compare various rest-frame mid-IR quantities as a function of time for our highly obscured (left) and less obscured (right) starburst simulations. At each time, we plot the median over viewing angle of each quantity. Vertical lines highlight the four characteristic times $t_{1}-t_{4}$ from Figure 1 , corresponding to pre-burst, peak starburst, peak AGN, and post-burst. The three curves in each panel represent the same models shown in the left column of Figure 1: we multiply the AGN luminosity deduced from the simulation by 0,1 , or 10 . In several rows, the gray solid (lined) bands subtend the $25 \%-75 \%$ range in the distribution of local ULIRGs (quasars) compiled by Veilleux et al. (2009). In the highly obscured case, the AGN is most dominant and most obscured at the time $t_{3}$, when the PAH emission $\left(r_{7.7}\right)$ and $\mathrm{Si}$ absorption $\left(r_{9.7}\right)$ ratios are minimized, in agreement with previous reasoning. The mid-infrared colors $f_{30} / f_{15}$ and $f_{15} / f_{5.7}$ also exhibit a weaker signal around this time, but this signal can be confused with the starburst phase $\left(t_{2}\right)$ for $f_{15} / f_{5.7}$, and depends non-linearly or not at all on the AGN strength with $f_{30} / f_{15}$. The more typical starburst represented in the right column is a marginal ULIRG, and its mid-infrared SED is relatively insensitive to the presence of the AGN which dominates at $1.4<t / \mathrm{Gyr}<1.8$.

(A color version of this figure is available in the online journal.)

To guide the discussion, we indicate the four times from the panels in Figure 1 with vertical lines for the highly obscured (left column) and less obscured (right column) mergers. The progenitors are still separated at time $t_{1}$, which precedes the period of final coalescence and peak $L_{\mathrm{IR}}$ by $\sim 200 \mathrm{Myr}$. The maximum SFR and AGN power occur at $t \sim t_{2}-t_{3}$, after the galaxies have merged, but the central sources are still largely obscured by dust. At $t_{4}$, up to $\sim 300$ Myr later, the SFR has plummeted but the AGN fraction is still elevated, and the remnants are on their way to being ellipticals.

Qualitatively, we find that our simulated mid-IR diagnostics behave largely as expected, but not always. Below, we address each of the diagnostics in turn.

The PAH strength, $r_{7.7}$, drops at the time of coalescence $\left(t_{2}-t_{3}\right)$, reaching its lowest values at $t_{3}$ when the AGN fraction is highest. Pre-coalescence, the PAH strength is weakest for the model with strongest AGN $(\mathrm{AGN} \times 10)$. Post-coalescence, the PAH strength again increases but does not return to its pre-coalescence levels. No significant "coalescence" dip in the PAH strength is seen in the less obscured case. The level of absorption by silicate grains, $r_{9.7}$, is greatest when the AGN is most luminous, which is also when the relative strength of the PAH emission is weakest. These extrema are relatively sharp and confined to the period of final coalescence at $\sim t_{2}-t_{3}$, although the AGN luminosity fraction remains elevated for $\approx 1$ Gyr. Overall, the shape of the PAH strength curve in both the highly obscured and less obscured cases does not bear a strong resemblance to the AGN fraction curve nor to the SFR curve.

In the highly obscured case, the $f_{15} / f_{5.7}$ slope is reddest when the AGN is most luminous, a somewhat counter-intuitive result since the $6 \mu \mathrm{m}$ continuum is generally believed to be enhanced by AGN torus emission. This result likely owes to the fact that the time when the AGN fraction is greatest corresponds to the time of greatest obscuration, when the $9.7 \mu \mathrm{m}$ silicate absorption feature is deepest. This obscuration would lead to significant absorption of the observed $6 \mu \mathrm{m}$ continuum, reddening the $f_{15} / f_{5.7}$ color. However, this may result from the simulations not having sufficiently clumpy ISM to allow for lines of sight directly to the AGN torus. This slope is also reddened at $t_{2}$, the time of peak SFR activity, as expected due to enhanced $\mathrm{H}$ II-region type emission. For the less obscured case, the $f_{15} / f_{5.7}$ color is reddened throughout the merger $\left(t_{1}-t_{4}\right)$, likely the result of the enhanced SFR, but lacks a sharper reddening at the time of coalescence. This supports our view that the "coalescence" reddening seen in the "highly obscured" case is a direct result of galaxy dust attenuation.

Similarly, the $f_{30} / f_{15}$ slope is effectively constant for the duration of the merger for the less obscured case, but is significantly reddened at the time of coalescence in the highly obscured case. At a given time, this slope is bluer when the AGN source is more luminous (e.g., $\mathrm{AGN} \times 10$ ), and in contrast to $f_{15} / f_{5.7}, f_{30} / f_{15}$ does not experience a sharp peak at $t_{3}$ for the most powerful AGN in the highly obscured merger.

Overall, our simulations indicate that the dependence of any of these mid-IR spectral diagnostics on the AGN fraction is timedependent and non-linear and is affected by the properties of the host galaxy. The dependence on the overall level of obscuration, as parameterized by the silicate absorption depth, is to some degree stronger. For example, these indicators vary much less when there is less obscuration.

When the highly obscured and less obscured simulations have the same AGN fraction, the highly obscured case shows much sharper coalescence-stage SED reddening and deepening of the silicate feature. These effects on the SED are therefore the direct result of the host galaxy dust attenuation. This supports earlier observational evidence (Lacy et al. 2007; Sajina et al. 2007; Juneau et al. 2011) that in at least some AGN-dominated, deep silicate absorption sources, the absorption could arise in the host galaxy rather than the AGN torus.

\subsection{Dust Model Dependence}

In this section, we address the effect of varying both the dust structure in the ISM (clumpy versus smooth) and its composition (MW-like versus SMC-like). In Section 2.4, we described the two ISM treatments we apply to the highly obscured merger. Broadly speaking, the "default ISM" case assumes the dust and gas associated with both hot and cold ISM phases are smoothly distributed. The "alternate ISM" assumes the cold phase gas is 


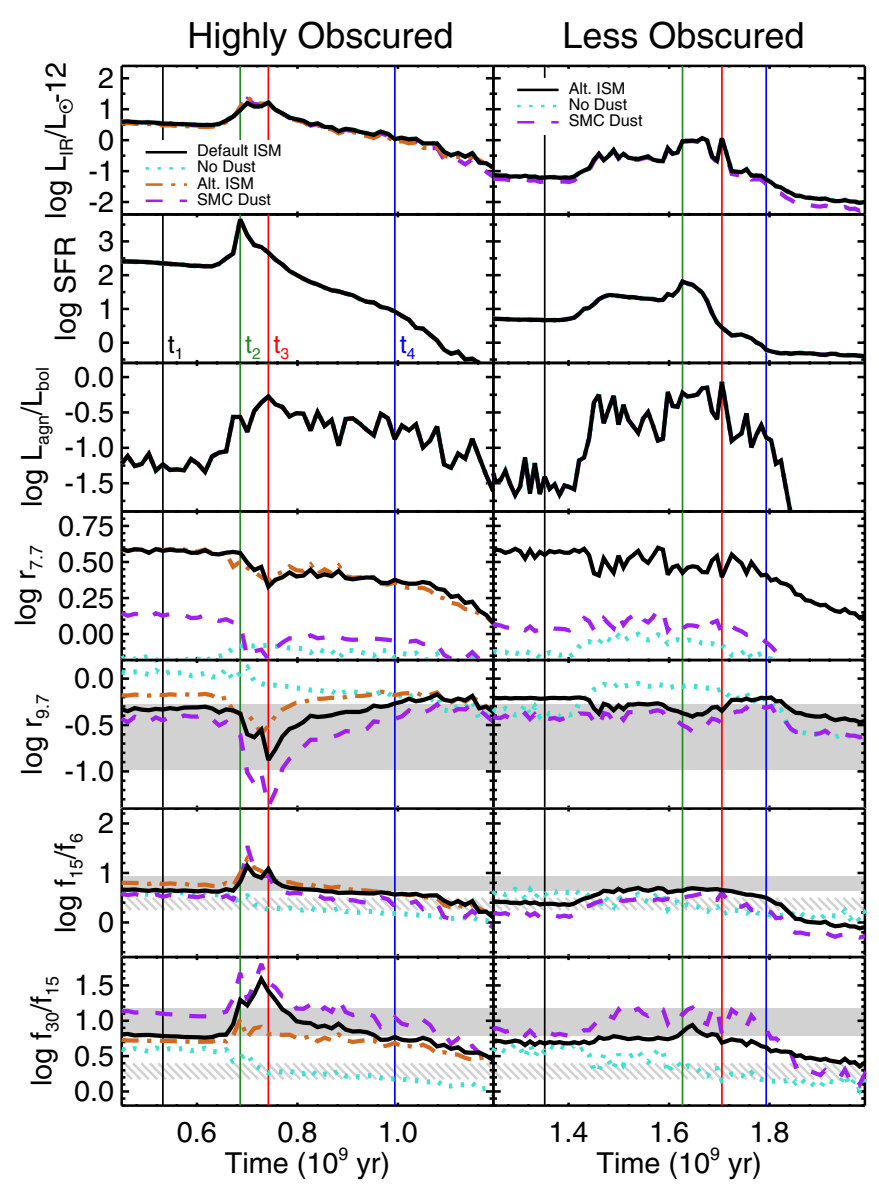

Figure 4. We compare the dust models in the same simulations and quantities as Figure 3. For both bursts, we show our fiducial models in solid black curves, the SMC dust grain model in the dashed purple curves, and the fiducial models without galaxy dust in the light blue curves. Vertical lines highlight the four characteristic times $t_{1}-t_{4}$ from Figure 1, corresponding to pre-burst, peak starburst, peak AGN, and post-burst. In the deeply obscured case, we also plot two alternate models: the SMC dust grain model and the clumpy ISM structure model. The powerful AGN signal in $r_{9.7}$ and $r_{7.7}$ at $t_{3}$ (Figure 3) can be replicated, at normal AGN strength, with the silicate-heavy SMC dust grain distribution. In several rows, the gray solid (lined) bands subtend the $25 \%-75 \%$ range in the distribution of local ULIRGs (quasars) compiled by Veilleux et al. (2009).

(A color version of this figure is available in the online journal.)

in clumps sufficiently dense to have a negligible volume filling factor, so that the dust it contains does not contribute to the overall attenuation. In Figure 4, we explore these effects on the mid-IR SEDs.

When switching to the alternate ISM assumption for the highly obscured simulation, the magnitude of $r_{9.7}$ is reduced by $\sim 0.3 \mathrm{dex}$, and the color $f_{30} / f_{15}$ by $\sim 0.7 \mathrm{dex}$, while $r_{7.7}$ and $f_{15} / f_{5.7}$ are mostly unchanged. In addition, the total IR luminosity (first row of Figure 4) is negligibly affected by this change. Therefore, the differences from this ISM structure assumption arise only because the source energy is redistributed in a different way, and not because of changes in the total amount of energy that is attenuated. This implies that there is less dust self-absorption in the alternate ISM model, which typically experiences less attenuation than the default case, so the source radiation that is reprocessed into the near-IR and mid-IR is not as often reprocessed again to longer wavelengths.

With SMC or MW dust, the IR SEDs at a given time during the starburst have similar slopes $f_{30} / f_{15}$ and $f_{15} / f_{5.7}$ (see also Figure 1). Furthermore, $L_{\mathrm{IR}}$, a measure of the amount of

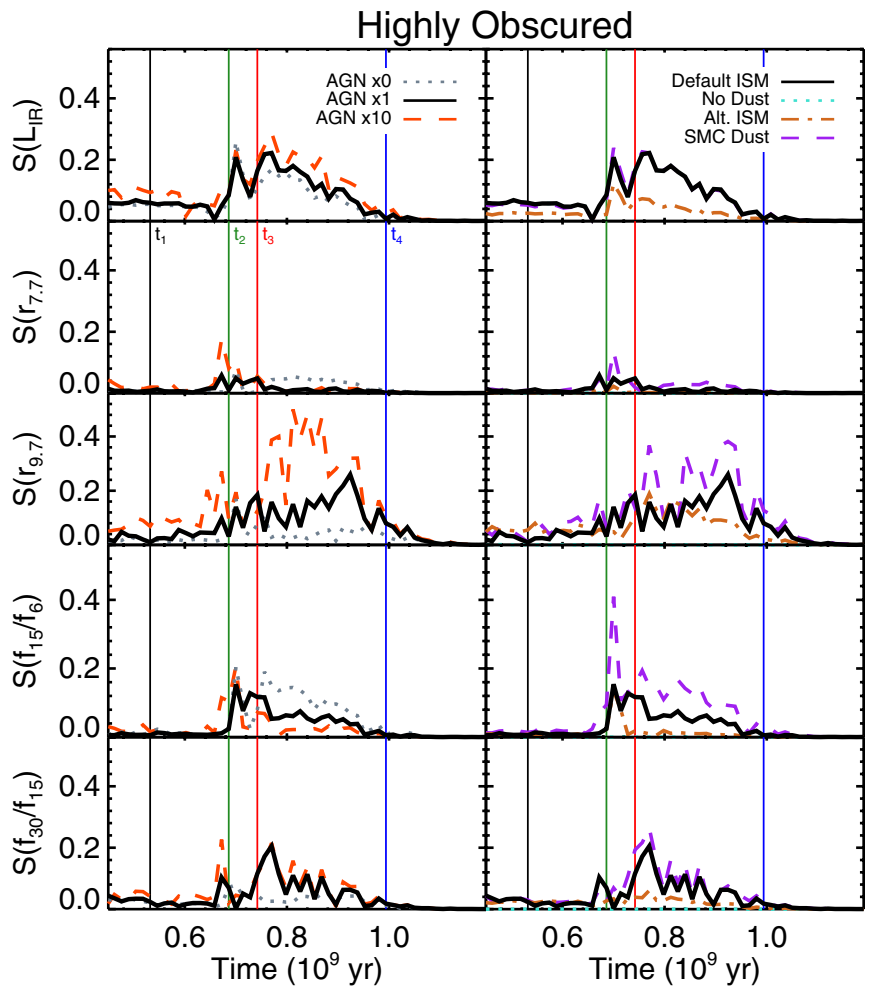

Figure 5. Scatter $S$, in dex, between viewing angles for the infrared quantities of the highly obscured case from Figures 3 and $4 . S$ is defined in the text of Section 4.3. Vertical lines highlight the four characteristic times $t_{1}-t_{4}$ from Figure 1, corresponding to pre-burst, peak starburst, peak AGN, and post-burst. The scatter $S$ is largest when the AGN is strongest, $\sim t_{3}-t_{4}$, because the obscuration varies significantly among the different sightlines to the AGN. The camera angle scatter for the less obscured burst (not shown) is much smaller, with $S$ always below 0.1 dex, and its median value below 0.05 dex.

(A color version of this figure is available in the online journal.)

attenuated light, is nearly identical in these cases. By contrast, the SEDs show very different spectral characteristics $r_{7.7}$ and $r_{9.7}$, reflecting the difference between $\mathrm{MW}$ and SMC grain composition. The SMC dust is assumed to contain relatively more silicate than carbonaceous grains, leading to virtually non-existent $\mathrm{PAH}$ emission $r_{7.7}$ and correspondingly stronger silicate absorption $r_{9.7}$.

\subsection{Viewing Perspective Dependence}

In addition to the (evolving) source SEDs, the observed SED depends on the evolving relative distributions of sources and dust. This evolution changes the relative obscuration of AGN and stellar sources, affecting the behaviors of mid-IR indicators depending on whether the luminosity is dominated by SF or AGN, because the AGN is generally much more highly obscured than the stellar component. In the Appendix, we use toy models to confirm and further examine the behavior of the simulated diagnostics.

Figure 5 shows the time-dependence of variation in the mid-IR quantities with viewing angle, focusing on the highly obscured merger. The camera angle scatter is much lower (median $S<0.05$ dex) for the less obscured merger. We show the same assumptions regarding AGN strength and ISM models from Figure 4, and we plot the scatter $S$, in dex, of the midIR quantities as a function of time. We define $S(q)$ to be the normalized median absolute deviation (MAD; e.g., Mosteller \& Tukey 1977; Beers et al. 1990) of the quantity $q$ among the seven SUNRISE viewing directions at a particular time during the 


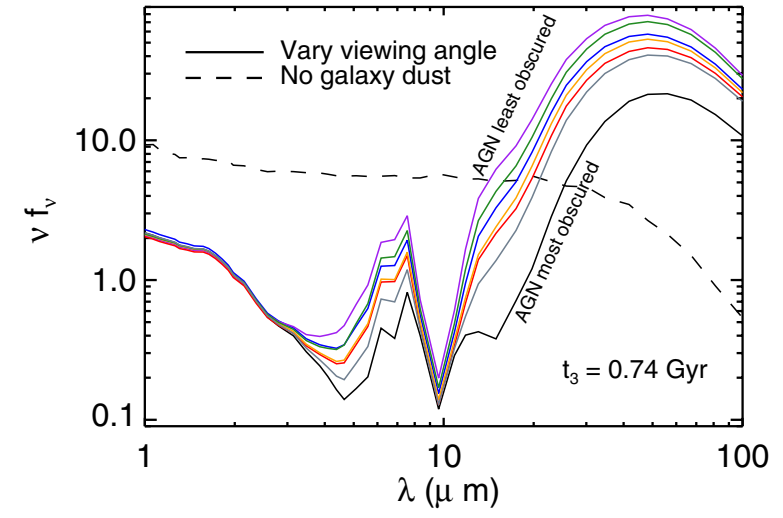

Figure 6. Infrared SEDs of the AGN $\times 1$ highly obscured burst simulation at time $t_{3}$ (AGN peak) observed from each of the seven viewing angles. The IR spectrum varies owing to the anisotropy of extremely optically thick material surrounding the central engine (starburst or AGN). Along a given direction, this dust absorbs IR light and reemits it into other lines of sight.

(A color version of this figure is available in the online journal.)

simulation. First, we calculate the median $M_{q}$ of $q$, and then the seven values $\left|q-M_{q}\right|$, the median of which we define to be the MAD of $q$. We define $S(q)=$ MAD/0.67 so that if $q$ is drawn from a Normal distribution with standard deviation $\sigma$, then $S$ is an unbiased estimator of $\sigma$.

At $t \lesssim t_{2}, S<0.1$ dex for all models, so the SED shape and spectral features are similar in all directions. Generally speaking, the scatter $S$ reaches a maximum $(\sim 0.2-0.4 \mathrm{dex})$ for conventional AGN diagnostics during $t_{3}$ to $t_{4}$. These times are also when the AGN luminosity fraction and IR luminosity are highest. Thus the increased viewing-angle scatter implies a large AGN contribution to the IR SED. This is because the AGN is effectively a point source, so the observed SED is sensitive to attenuation along a single line of sight, which can vary widely. Moreover, the sudden change in the extinction curve at $9.7 \mu \mathrm{m}$ causes $r_{9.7}$ to have an exaggerated dependence on the lineof-sight attenuation. $r_{7.7}$ experiences much less viewing angle scatter than $r_{9.7}, S\left(r_{7.7}\right) \sim 0.0$ versus $S\left(r_{9.7}\right) \sim 0.2$ dex, because the extinction is the same for both the PAH features and $7.7 \mu \mathrm{m}$ continuum. Turning off AGN emission leads to essentially no viewing angle variation in either quantity.

Figure 6 presents the SED from seven viewing angles at time $t_{3}$ (peak AGN) for our highly obscured merger, with AGN $\times 1$ and fiducial ISM models. This gives us a clearer physical picture of what is driving changes to the mid-IR diagnostics during coalescence. The generally higher level of obscuration at these times leads to the enhanced silicate absorption, causing the dip in median $r_{9.7}$ at $t_{3}$ (Figure 3). However, at a fixed time during this period of very high attenuation, like the one shown in Figure 6, the silicate absorption feature is stronger along lines of sight in which the AGN is less obscured. In other words, the silicate feature only correlates positively with AGN attenuation until the level of attenuation is so extreme that the emergent stellar SED dominates the mid-IR emission associated with the AGN. This phenomenon is derived in the Appendix for a toy model of $r_{9.7}$.

\subsection{Intrinsic AGN Emission Dependence}

In Figure 7, we consider the effect of the intrinsic mid-IR AGN SED shape on the final observed SED. We show the manually boosted model $(\mathrm{AGN} \times 10)$ in order to maximize the possible effect. We demonstrate that the intrinsic AGN mid-IR

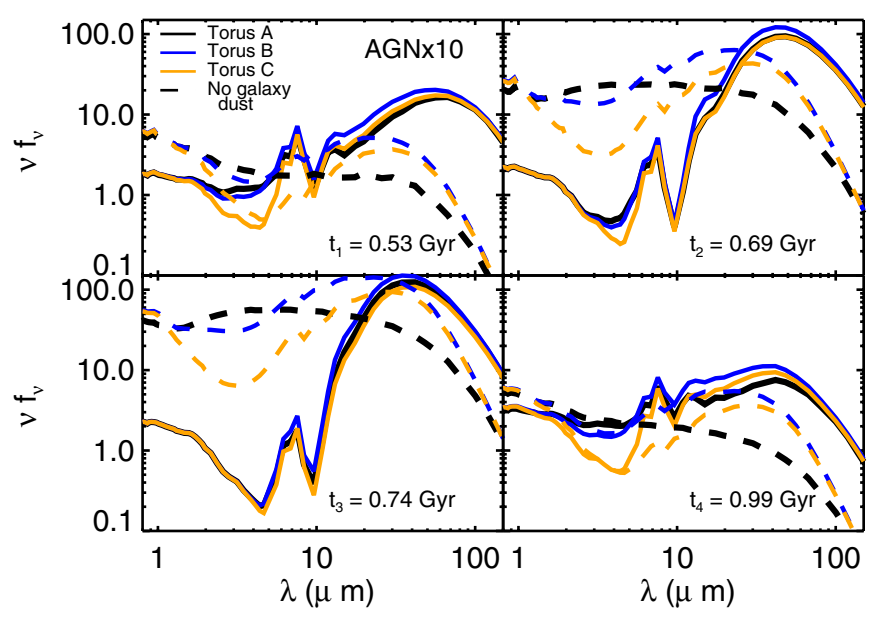

Figure 7. We explore the sensitivity of the final infrared SED to the assumed intrinsic AGN (or AGN+torus) spectrum, focusing on the highly obscured simulation. In this figure, we use the manually boosted AGN $(\times 10)$ input SED, and so the effect sizes shown here should be considered upper limits. The solid lines show the emergent SED from an example viewing direction with three assumptions about the intrinsic AGN SED. The dashed lines show the intrinsic stellar+AGN source emission before the dust RT calculation. The black lines use the mean templates from Richards et al. (2006a), while the blue and yellow lines use $i=0^{\circ}$ and $i=90^{\circ}$ clumpy torus models from Nenkova et al. (2008), respectively. The choice of intrinsic AGN SED matters least at $t_{3}$, when the SMBH's contribution to the total luminosity is largest. The AGN fraction at times $t_{2}-t_{4}$ are all $\gtrsim 70 \%$ and yet the appearance of the mid-IR SED changes dramatically over these several $10^{8} \mathrm{yr}$. The influence of the intrinsic AGN spectrum on the final SED at $5 \mu \mathrm{m}$ increases from $\sim 0$ dex at $t_{3}$ to $\sim 1$ dex at $t_{4}$. (A color version of this figure is available in the online journal.)

spectrum has no impact on the observed mid-IR SED at $t_{2}-t_{3}$ of the highly obscured merger, during the starburst and AGN peak at final coalescence when the central densities are highest. We use our default AGN SED, the template by Hopkins et al. (2007c), as well as two templates by Nenkova et al. (2008). These templates reflect obscuration by clumpy tori inclined by $i=0^{\circ}$ and $i=90^{\circ}$, spanning the range of derived mid-IR torus SED shapes.

With our default ISM assumption, dust obscures this central source at wavelengths as long as $\lambda \sim 20-50 \mu \mathrm{m}$. Thus the flux at $\lambda \sim 2-5 \mu \mathrm{m}$, conventionally attributed to AGN tori, only indicates direct AGN emission when the central source is sufficiently unobscured (e.g., $t_{1}$ and $t_{4}$ ). In the less obscured simulation, the AGN torus SED has a large effect throughout the merger, as seen by the near-IR slopes of the SEDs in the left column of the "Less Obscured" panels in Figure 1.

\section{OBSERVATIONAL COMPARISON}

\subsection{Two-dimensional Diagnostics}

In Section 4, we presented the time evolution of the observed mid-IR indicators and noted that their dependence on $L_{\text {agn }} / L_{\text {bol }}$ is both time-dependent and non-linear. We showed that our midIR spectral diagnostics strongly depend on the dust model and can be highly scattered as a function of viewing angle. How could we hope to observationally discern the level of AGN in a given galaxy based on its mid-IR spectrum, given that typically no information on the merger stage is available, the dust model is even less constrained, and we will never have more than one viewing perspective? Here we attempt to do just that by using the simulation results as a diagnostic tool.

In Figure 8, we present mid-IR diagnostic diagrams that have been used in the literature for the purpose of disentangling 


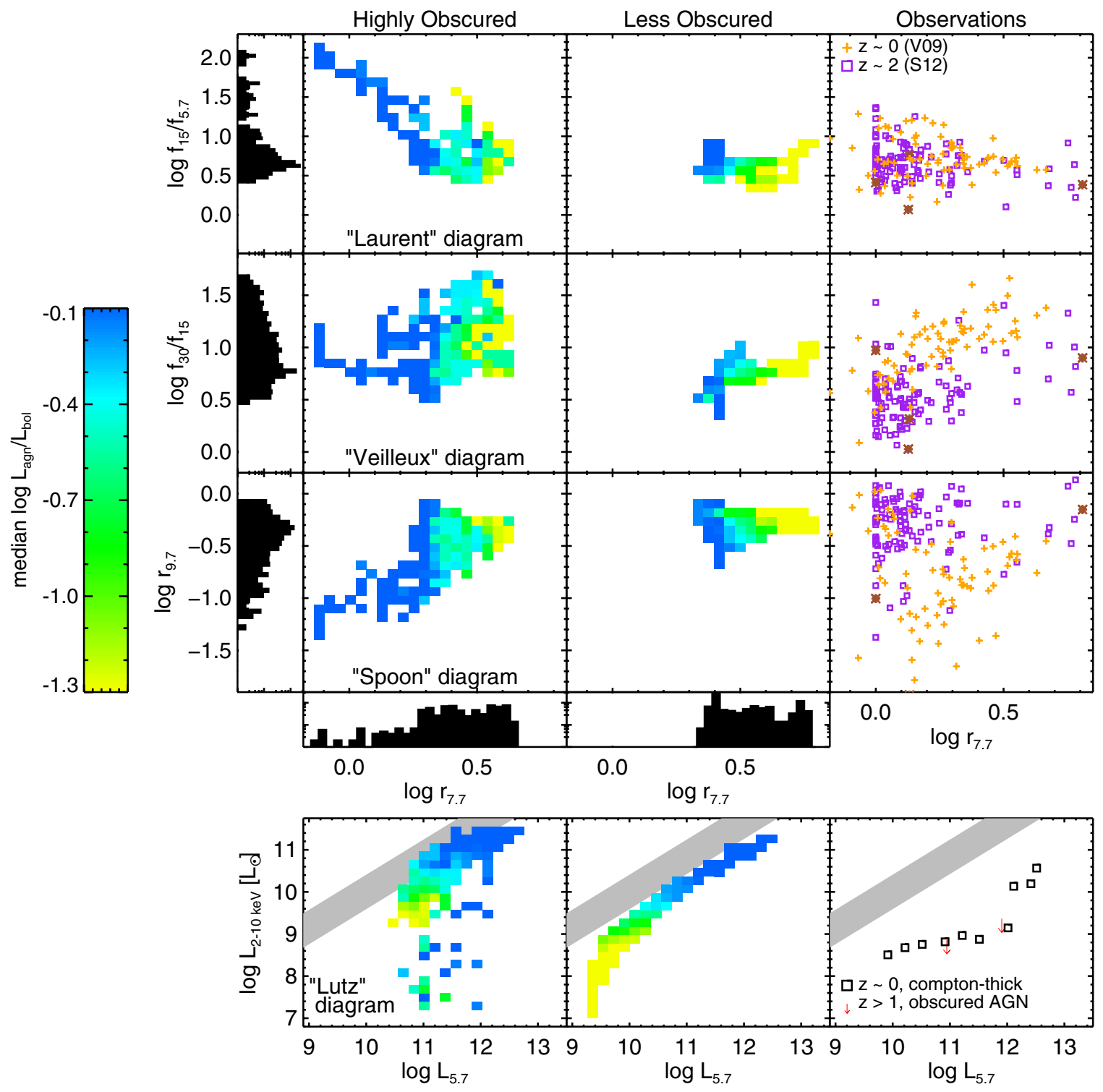

Figure 8. We compare the two simulations to observed samples of ULIRGs. The four rows each present an example diagnostic diagram that has been examined in the literature. Within each row, the left plot shows the "highly obscured" model points colored according to the median AGN fraction $L_{\text {agn }} / L_{\text {bol }}$ within each grid cell: see the text for a description of this procedure, and refer to the color bar at the left. The middle panel of each row shows the same mapping for the "less obscured" simulation. We employ the fiducial ISM settings for these panels, in particular MW dust, and include all points between $t_{1}-t_{4}$ from the AGN $\times 10$, AGN $\times 1$, and AGN $\times 0$ models as a means of exploring the sensitivities of these SEDs to changes in modeled AGN strength. We show a histogram around the edges of the first three columns to highlight the relative amount of time spent by the simulations in each region. We find that dark squares span almost the entire $y$ axis in the left column of the top three rows, implying that powerful AGN can occupy any value of these diagnostics in our two merger models. The right panel in each row plots observed local V09 and high- $z$ S12 ULIRGs. When the $r_{9.7}$ feature is saturated, we plot a brown asterisk. In the "Lutz diagram" in the bottom row, the gray shaded region shows the $1 \sigma$ range of intrinsic X-ray-to-mid-IR ratio for Type 1 AGN from Bauer et al. (2010).

(A color version of this figure is available in the online journal.)

the role of AGN and starburst activity in dusty galaxies. Specifically, using our definitions of the PAH strength, silicate feature depth, and mid-IR colors, we present slightly modified versions of the "Laurent" diagram (Laurent et al. 2000), the "Spoon" diagram (Spoon et al. 2007), the "Veilleux" diagram (Veilleux et al. 2009), and the "Lutz" diagram (Lutz et al. 2004). The last diagram presents the relation between the $6 \mu \mathrm{m}$ luminosity and X-ray luminosity for unobscured or mildly obscured AGNs. The left-hand column of Figure 8 presents the highly obscured simulation, the middle column presents the less obscured simulation, and the right-hand panel presents real galaxy data that we discuss in more detail in the following section.
For the simulation columns in Figure 8, we plot the IR quantities in the following way. Each panel is divided into a grid of $25 \times 25$ bins, each of which contains zero or more simulated points. These points include all seven viewing angles at each timestep in $t_{1}-t_{4}$, which are sampled every $10^{7} \mathrm{yr}$. We use our fiducial ISM settings and plot the three AGN strengths $(\times 10$, $\times 1$, and $\times 0$ ) in order to span a full range of $L_{\mathrm{agn}} / L_{\mathrm{bol}}$ in our experiments. Bins containing zero of these points are white, and bins with one or more point are shaded according to the median value of $L_{\mathrm{agn}} / L_{\mathrm{bol}}$ among the points it contains, as indicated by the color bar: darker/blue regions have $L_{\mathrm{agn}} / L_{\mathrm{bol}} \sim 1$, while lighter/yellow regions have $L_{\mathrm{agn}} / L_{\text {bol }} \lesssim 0.1$. We plot histograms (on a logarithmic scale) along the edges of the 
first three rows to show the relative timescales of the model populations giving rise to these diagrams.

We caution that our plotting and selection here are not meant to directly characterize the observed populations of ULIRGs that we plot in the right column; these are included primarily as a reference and plausibility check. Instead, from this coding we seek to identify which regions, if any, isolate powerful AGNs during these mergers, regardless of the length of time the observed properties may be visible. A larger sample of models will be needed in order to fully constrain the expected observed distribution of $L_{\mathrm{agn}} / L_{\mathrm{bol}}$ at each location in these diagrams, but we can begin to highlight some basic trends implied by the simulations.

\subsection{Comparison Between Models and Data}

Veilleux et al. (2009) analyzed the standard evolutionary scenario for ULIRGs (Sanders et al. 1988), which broadly speaking proceeds through three stages. In "Stage 1," the IR emission of a gas-rich merger is dominated by SF, leading to large PAH EWs and relatively small silicate absorption EWs. As the merger proceeds to "Stage 2," $r_{9.7}$ increases as the central source is buried in the gas-rich starburst, and the PAH emission decreases relative to the IR continuum that is boosted by the AGN, which remains sub-dominant to the starburst. Finally, at "Stage 3," the silicate absorption and PAH emission both subside as the starburst fades, the obscuring medium is cleared or consumed, and the ULIRG is completely or nearly AGNdominated. Overall, Veilleux et al. (2009) concluded that AGNs contribute $\sim 35 \%-40 \%$ to the IR luminosity of ULIRGs, and that ULIRGs experience significant scatter from this general pattern.

In Figure 8, the highly obscured model points roughly fill the space spanned by observed sources. Our procedure does not mandate such consistency, and so we interpret this as one important check of the modeling technique. In particular, ignoring other diagnostics (such as SED slopes), this simulation reproduces the observed spread in $r_{9.7}$ and $r_{7.7}$. In Table 2, we summarize a few numerical results of our deeply obscured simulation. We divide the merger into three stages, $t_{1}-t_{2}$ (pre-coalescence), $t_{2}-t_{3}$ (coalescence), and $t_{3}-t_{4}$ (post-coalescence), and report the median values of $L_{\mathrm{agn}} / L_{\mathrm{bol}}, r_{9.7}$, and $r_{7.7}$ calculated in those periods.

Generally, the SED properties along the model timeline follow those of the merger stages by V09, albeit with an expectedly high amount of scatter. Local ULIRGs have a large amount of silicate absorption $\left(r_{9.7} \sim 0.7\right)$, suggesting they may correspond to the modeled points at $t_{2}<t<t_{3}$ where $r_{9.7}$ peaks. However, the AGN fractions in models depend sensitively on the dust model and AGN strength choices, and so it is unclear how well they may correspond to the $\sim 30 \%$ reported in V09. This value is consistent with our $\mathrm{AGN} \times 1, \mathrm{MW}$ dust simulation, but is much lower than the $\sim 70 \%$ from the "combined" model set, in which each of the AGN strengths are given equal weight. The more realistic choice is uncertain, but the AGN $\times 1$ case has trouble reproducing observed objects with $r_{9.7}<-1$. However, SMC dust with lower AGN strengths can also provide that feature, and observations suggest that SMC-like dust may be more appropriate during bright starburst or AGN phases, as small grains are preferentially depleted (Gordon et al. 1997; Hopkins et al. 2004). This may imply that SMC dust is more appropriate to use in this situation; in any case, it highlights the difficulty in determining the path of the AGN fraction in such objects if their dust grain populations are changing significantly.
Table 2

Selected Diagnostics, Deeply Obscured Case

\begin{tabular}{|c|c|c|c|}
\hline Time Range ${ }^{a}$ & $\log r_{7.7^{b}}$ & $\log r_{9.7} b$ & $\log L_{\mathrm{agn}} / L_{\mathrm{bol}}^{\mathrm{b}}$ \\
\hline \multicolumn{4}{|c|}{$\mathrm{AGN} \times 0, \mathrm{MW}$ dust } \\
\hline$t 1<t<t 2$ & 0.62 & -0.31 & 0.00 \\
\hline$t 2<t<t 3$ & 0.56 & -0.40 & 0.00 \\
\hline$t 3<t<t 4$ & 0.52 & -0.37 & 0.00 \\
\hline \multicolumn{4}{|c|}{$\mathrm{AGN} \times 1, \mathrm{MW}$ dust } \\
\hline$t 1<t<t 2$ & 0.57 & -0.33 & 0.06 \\
\hline$t 2<t<t 3$ & 0.46 & -0.62 & 0.33 \\
\hline$t 3<t<t 4$ & 0.39 & -0.43 & 0.26 \\
\hline \multicolumn{4}{|c|}{$\mathrm{AGN} \times 1, \mathrm{SMC}$ dust } \\
\hline$t 1<t<t 2$ & 0.12 & -0.44 & 0.06 \\
\hline$t 2<t<t 3$ & -0.13 & -1.09 & 0.33 \\
\hline$t 3<t<t 4$ & -0.03 & -0.70 & 0.26 \\
\hline \multicolumn{4}{|c|}{$\mathrm{AGN} \times 10, \mathrm{MW}$ dust } \\
\hline$t 1<t<t 2$ & 0.40 & -0.37 & 0.43 \\
\hline$t 2<t<t 3$ & 0.04 & -0.95 & 0.86 \\
\hline$t 3<t<t 4$ & 0.30 & -0.40 & 0.81 \\
\hline \multicolumn{4}{|c|}{ Combined, MW dust } \\
\hline$t 1<t<t 2$ & 0.57 & -0.32 & 0.36 \\
\hline$t 2<t<t 3$ & 0.45 & -0.65 & 0.70 \\
\hline$t 3<t<t 4$ & 0.39 & -0.38 & 0.58 \\
\hline
\end{tabular}

Notes.

a These time ranges are $0.154,0.056$, and $0.252 \times 10^{9} \mathrm{yr}$ long, respectively.

b Median values.

In Figure 8, swaths of strong and weak AGN exist, but most of these projections do not isolate AGN in simple ways. For example, while it is true that sources with strong starbursts and weak AGNs typically have stronger PAH emission and redder $f_{30} / f_{15}$ as discussed in Veilleux et al. (2009), strong AGNs occupy all values of $f_{30} / f_{15}, f_{15} / f_{5.7}$, and $r_{9.7}$. Furthermore, the PAH strength depends sensitively on the dust model we assumed (see Figure 4 ), and the $r_{7.7}$ values of low $L_{\text {agn }} / L_{\text {bol }}$ points depend on the obscuration level of the simulation.

In addition, we compare the relative fluxes emerging at hard $\mathrm{X}$-ray and mid-IR wavelengths in the bottom row of Figure 8. We compute model X-ray fluxes as in Section 2.5. Bauer et al. (2010) found that $z>1$ ULIRGs that are otherwise thought to be AGN-dominated (with low $r_{7.7}$ ) are undetected at X-ray wavelengths, suggesting that they are at least mildly Comptonthick. Moreover, they presented Compton-thick examples from the literature at $z \sim 0$, which lie $\sim 2$ dex below the relation for less obscured AGNs (cf. Hönig et al. 2010). Many model galaxies with powerful AGNs lie in the same region of this diagram, and are reflected in the dark blue squares mixed in with the lighter squares at $L_{6} \sim 10^{11}-10^{12} L_{\odot}$. These are the same sources in the low- $r_{7.7}$ (high $r_{9.7}$ ) tail of dark points in the upper panels, implying that this class of Compton-thick sources is a short-lived phase of our highly obscured model. The bulk of the simulation points have X-ray fluxes $\sim 0.5$ dex below the gray Type 1 AGN band, reflecting our choice to not analyze completely unobscured models.

There are some properties for which the model values are not realized by observed sources. Generally speaking, the differences are most apparent in the mid-IR SED slopes $f_{30} / f_{15}$ and $f_{15} / f_{5.7}$. The most AGN-dominant model points are $\approx 0.5$ dex redder in $f_{15} / f_{5.7}$ than any observed object. In bulk, the model $f_{30} / f_{15}$ values are larger by $\approx 0.3$ dex than the 


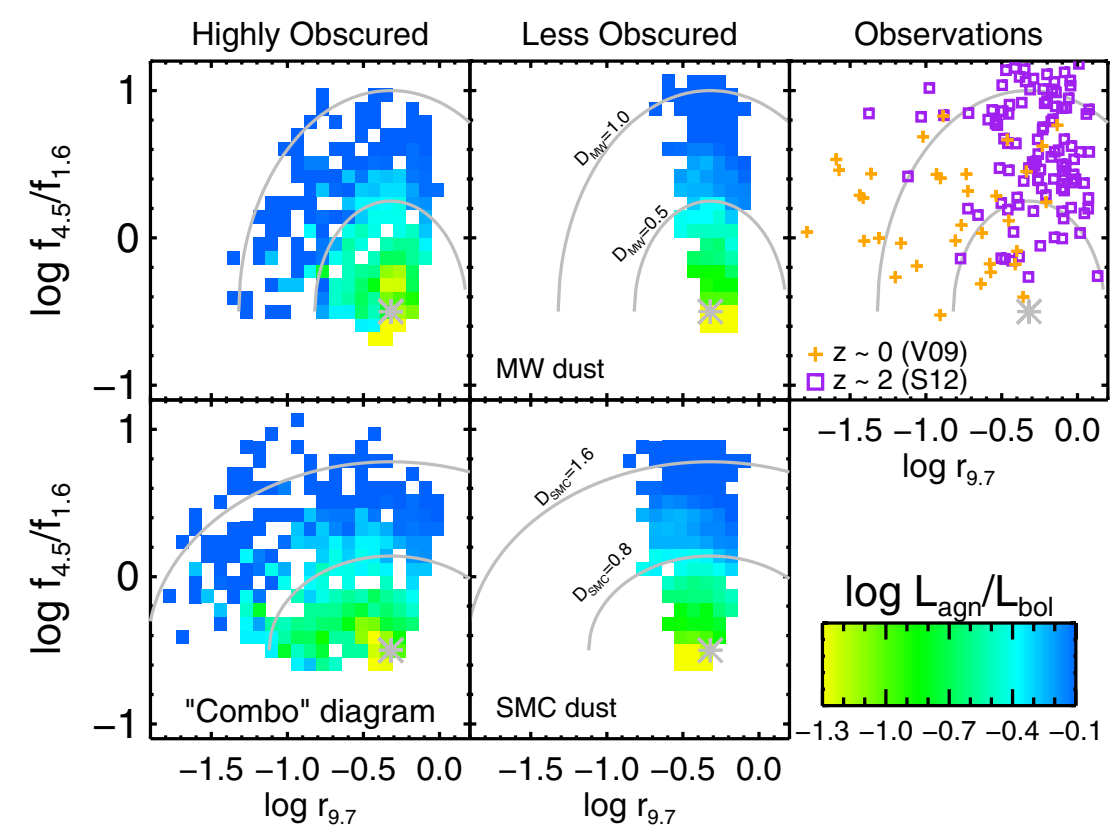

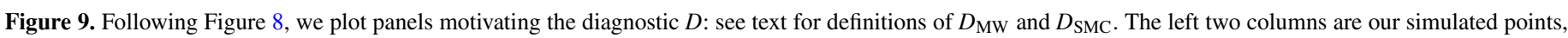

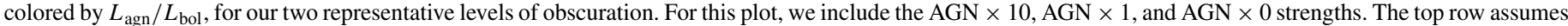

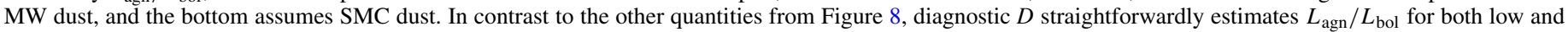

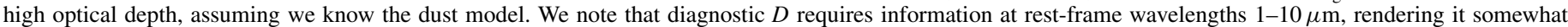
impractical to measure using any single current instrument. However, diagnostic $D$ will be accessible to the James Webb Space Telescope for sources at $z \lesssim 1$.

(A color version of this figure is available in the online journal.)

observed distribution, and there are few model galaxies with $\log r_{9.7} \sim 0$ and $\log r_{7.7} \sim 0$, in contrast to those observed. However, this is sensitive to our dust grain model (Section 6.2), and may also reflect our not having modeled AGNs with no galaxy obscuration. Our calculations are necessarily uncertain, so in Section 6.3 below, we summarize some of the theoretical limitations and potential implications of these mismatches.

As we have seen in Section 4, when sufficiently obscured, a powerful AGN has an SED shape that is the same as a dusty pure starburst (see also Younger et al. 2009; Narayanan et al. 2010a). Figure 8 shows high $L_{\text {agn }} / L_{\text {bol }}$ squares occupying a large fraction of the $r_{7.7}, r_{9.7}, f_{30} / f_{15}$, and $f_{15} / f_{5.7}$ values. We also saw that the model SEDs can change drastically on timescales $\sim 10^{8} \mathrm{yr}$. A further challenge is that the implied $L_{\mathrm{agn}} / L_{\mathrm{bol}}$, given a set of SED properties, depends sensitively on assumptions about the dust model and AGN strength. An ideal indicator is one that identifies powerful AGNs regardless of the host galaxy's content or state. An example is hard X-ray emission which can penetrate even very large optical depths, and in Section 6, we use our simulations to suggest other ideal indicators that have not been easily accessible with current data, but may be useful for future mid-IR studies with the JWST.

\section{IMPLICATIONS}

\subsection{An Ideal Indicator of $L_{a g n} / L_{b o l}$}

Ultimately, we seek an indicator that can be used to determine the AGN contribution to IR-luminous galaxies across the full range of obscuration by galaxy dust. Such a tool could simplify efforts to accurately track the growth of SMBHs and assess their impact on the ISM in diverse and evolving host galaxies.

Near-infrared colors effectively identify AGNs when their IR SED resembles a power-law, leading to enhanced emission beyond the stellar bump at $\gtrsim 2 \mu \mathrm{m}$. This yields a high value of our example near-IR color $f_{4.5} / f_{1.6}$, for which the numerator is a proxy for torus emission and the denominator is a proxy for stellar mass. Such techniques have been used to successfully identify high-redshift AGN-dominated ULIRGs using Widefield Infrared Survey Explorer photometry (e.g., Stern et al. 2012; Eisenhardt et al. 2012; Wu et al. 2012). This nearIR reddening operates in powerful AGNs for a wide variety of attenuation. However, as we saw in Figure 7, on short timescales, the near-IR SED shape can change from a power-law AGN to one that resembles a starburst-dominated ULIRG when the obscuration increases drastically. In these high-obscuration situations, $r_{9.7}$ is sensitive to the AGN power, and so combining these indicators into a generic diagnostic may be feasible.

In Figure 9, we plot two-dimensional diagrams with our nearIR color $f_{4.5} / f_{1.6}$ on the $y$-axis and $r_{9.7}$ on the $x$-axis. We have included our fiducial ISM assumptions for each mergers and all three AGN strengths: $A G N \times 10, A G N \times 1$, and $A G N \times$ 0 . We note that these settings are chosen by hand in order to more broadly span the range of $L_{\mathrm{agn}} / L_{\mathrm{bol}}$ produced by our calculations, and are justified by the uncertainty inherent in the simulation methods and limited range of cosmologically motivated models we consider (see, e.g., Section 2.4). Therefore, these model diagrams do not directly correspond with any realistic ULIRG populations; we plot two samples in the right panel primarily as a reference.

The light/yellow shaded region corresponds to low AGN emission $\left(L_{\mathrm{agn}} / L_{\mathrm{bol}} \lesssim 0.1\right)$ and is confined to a small region $(\sim 0.4$ by 0.4 dex $)$ of this parameter space centered at $\left(\log r_{9.7}, \log f_{4.5} / f_{1.6}\right) \approx(-0.32,-0.5)$. In both the highly and less obscured simulations, the value $\log f_{4.5} / f_{1.6} \sim-0.5$ corresponds to the SED shape of purely stellar emission, which can be seen in the gray dotted curves in Figure 1 at $1 \mu \mathrm{m}<\lambda<5 \mu \mathrm{m}$. The value $\log r_{9.7} \sim-0.32$ appears to be the typical SED produced by ordinary (non-bursting) SF in our simulations. Numerically, this value may be set by our simplistic continuum estimate (Section 3.1), which is likely affected to some extent 
by the nearby PAH features. Both of these "zeropoints" do not depend strongly on the dust model, and are roughly the same in the highly and less obscured mergers.

Away from this point, the median AGN fraction varies in a semi-regular pattern. A redder $f_{4.5} / f_{1.6}$ implies a contribution at $4.5 \mu \mathrm{m}$ from the relatively brighter AGN source, as expected from conventional hot dust/torus interpretations. The absorption diagnostic $r_{9.7}$ does not vary strongly in the less obscured case, as we have seen in Section 4.1, and so the locus of increasing $L_{\text {agn }} / L_{\text {bol }}$ traces roughly a vertical line in the second column of Figure 9.

By contrast, in the highly obscured simulation, a significant area of the $\log r_{9.7} \lesssim-0.5$ regions of the diagram is occupied by squares with $L_{\text {agn }} / L_{\text {bol }} \gtrsim 0.1$. In the lower left quadrant of each panel in the left column of Figure 9, the dust column is large enough to produce a significant silicate absorption feature and to attenuate the near-IR continuum associated with AGN-heated dust, leading to $\log f_{4.5} / f_{1.6} \lesssim 0$. In the upper left quadrant, there are several squares with both a strong silicate feature and a red near-IR continuum. At a fixed $L_{\mathrm{agn}} / L_{\mathrm{bol}}$, these two quantities are correlated in the sense that increased silicate absorption implies more attenuation of the near-IR AGN-heated continuum relative to the starlight, giving a bluer $f_{4.5} / f_{1.6}$, leading to the slanted or semi-elliptical pattern in the areas of constant shading $\left(L_{\mathrm{agn}} / L_{\mathrm{bol}}\right)$ in Figure 9 . These qualities are the same when switching to the "alt. ISM" model for the highly obscured merger (not shown).

We seek to summarize these trends and estimate $L_{\mathrm{agn}} / L_{\mathrm{bol}}$ directly from these variables. Visually, the areas of constant $L_{\text {agn }} / L_{\text {bol }}$ form a semi-elliptical pattern that can be approximated by an analytic formula to potentially estimate $L_{\mathrm{agn}} / L_{\mathrm{bol}}$ with low scatter. For MW dust, we consider the formula

$$
D_{\mathrm{MW}}=\sqrt{\left(\log r_{9.7}+0.32\right)^{2}+\left(\left[\log \frac{f_{4.5}}{f_{1.6}}+0.5\right] \frac{2}{3}\right)^{2}} .
$$

The effectiveness of combination $D_{\mathrm{Mw}}$ is evident in the top row of Figure 9: the map between color/shading $\left(L_{\mathrm{agn}} / L_{\mathrm{bol}}\right)$ and these axes, $f_{4.5} / f_{1.6}$ and $r_{9.7}$, appears to be a well defined and slowly varying function of few parameters. In the Appendix, we confirm the rough behavior of this diagnostic from toy models.

We caution that we have analyzed only a handful of specially chosen models: $\mathrm{AGN} \times 10, \mathrm{AGN} \times 1$, and $\mathrm{AGN} \times 0$ in two mergers that likely occupy two very different extremes of the ULIRG population. Therefore, the shaded points in Figures 8 and 9 are likely to change with a larger and more representative sample of models. Nevertheless, it is encouraging that a clear trend in the $f_{4.5} / f_{1.6}-r_{9.7}$ plane exists for both our highly and less obscured mergers (in contrast to the diagrams in Figure 8), and for either the default or alternate ISM model in the highly obscured merger. This can be understood as a result of the relatively simple ways that the two axes of Figure 9 reflect AGN-associated emission and its obscuration. In Section 6.2, we show that this visual trend we identify in Figure 9 defines a tight relationship between $L_{\mathrm{agn}} / L_{\mathrm{bol}}$ and $D_{\mathrm{MW}}$ at all times $t_{1}-t_{4}$ when the dust model is constrained.

\subsection{Constraining Dust Properties}

However, we note that shape of the trend in the $f_{4.5} / f_{1.6}-r_{9.7}$ plane depends on our dust model: the bottom row of Figure 9 shows the same simulation using SMC dust instead of MW dust. Importantly, a nice mapping in the above sense still exists, it is just skewed toward higher values of $r_{9.7}$. Therefore, we can

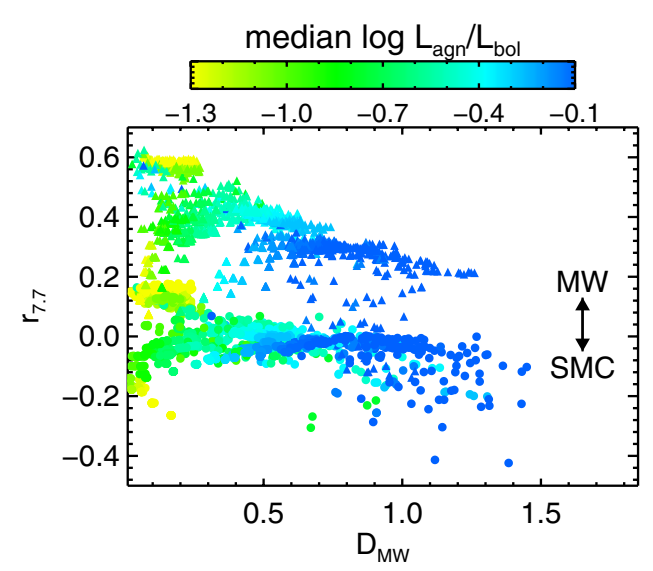

Figure 10. PAH strength vs. our combination diagnostic $D_{\mathrm{MW}}$, which contains information about obscuration $r_{9.7}$ and near-IR color, providing a rough estimate of $L_{\text {agn }} / L_{\text {bol }}$ at both low and high mid-IR optical depths. If we have incorrectly assumed MW dust when the appropriate model is more like the SMC dust, then Figure 9 implies that the $D_{\mathrm{MW}}-L_{\mathrm{agn}} / L_{\mathrm{bol}}$ relation will be skewed and have a large scatter. However, at fixed high $D_{\mathrm{MW}}$, the PAH strength can roughly select the appropriate dust model, and therefore the appropriate predictor of $L_{\text {agn }} / L_{\text {bol }}$ : $D_{\mathrm{MW}}, D_{\mathrm{SMC}}$, or an interpolation. For this plot, we have included the AGN $\times$ 1 and AGN $\times 10$ strengths for the default ISM model of the highly obscured merger.

(A color version of this figure is available in the online journal.)

define a similar diagnostic,

$$
D_{\mathrm{SMC}}=\frac{5}{8} \sqrt{\left(\log r_{9.7}+0.32\right)^{2}+\left(\left[\log \frac{f_{4.5}}{f_{1.6}}+0.5\right] \frac{5}{4}\right)^{2}},
$$

centered around the same point as $D_{\mathrm{MW}}$, but with a different axis ratio and scaling.

In order to select between these estimators, we must first constrain the dust properties; in other words, we must jointly constrain the dust and $L_{\mathrm{agn}} / L_{\mathrm{bol}}$. One option is to use the PAH emission: assuming SMC dust, $r_{7.7} \sim 0$ for all model points instead of $\sim 0.3$ for MW dust (see Figure 4 ). We demonstrate this procedure in Figure 10: while $D_{\text {Mw }}$ does not yield a low-scatter predictor of $L_{\text {agn }} / L_{\text {bol }}$ when applied to a system with SMC dust, it does broadly select stronger AGN. Moreover, at $D \gtrsim 0.2$, sources with these different dust models separate based on their PAH strength, $r_{7.7}$, permitting either a direct choice between the formulae for $D_{\mathrm{MW}}$ and $D_{\mathrm{SMC}}$ or an intermediate case. This suggests the following procedure:

1. Estimate $D_{\text {Mw }}$.

2. Estimate the PAH strength (here, $r_{7.7}$ ).

3. Select dust model $X$ based on the value obtained in step 2, by, e.g., checking whether at the $D_{\mathrm{MW}}$ value, $r_{7.7}$ lies closer to the MW or SMC points.

4. Calculate $D_{\mathrm{X}}$ and use it to estimate $L_{\mathrm{agn}} / L_{\mathrm{bol}}$.

With a known dust model, diagnostic $D$ robustly predicts $L_{\text {agn }} / L_{\text {bol }}$ regardless of the physical conditions of the ULIRG source. We compare the ability of diagnostics $D_{\mathrm{MW}}$ and $D_{\mathrm{SMC}}$ to predict $L_{\mathrm{agn}} / L_{\mathrm{bol}}$ to that of hard X-ray fluxes in Figure 11.

Using a least absolute deviation method, we fit the relation $L_{\mathrm{agn}} / L_{\mathrm{bol}}=a+b Q$ for three quantities $Q\left(D_{\mathrm{MW}}\right.$ with $\mathrm{MW}$ dust, and both $D_{\mathrm{MW}}$ and $D_{\mathrm{SMC}}$ with SMC dust) and each of our two ISM models for the highly obscured simulation, and compile the results in Table 3 . To measure the scatter in the correlations' residuals, we used the same scale estimator $S$ defined in Section 4.3, which is generally insensitive to outliers. In both cases, $L_{2-10 \mathrm{keV}}$ and each $D$ predict $L_{\text {agn }} / L_{\text {bol }}$ with 


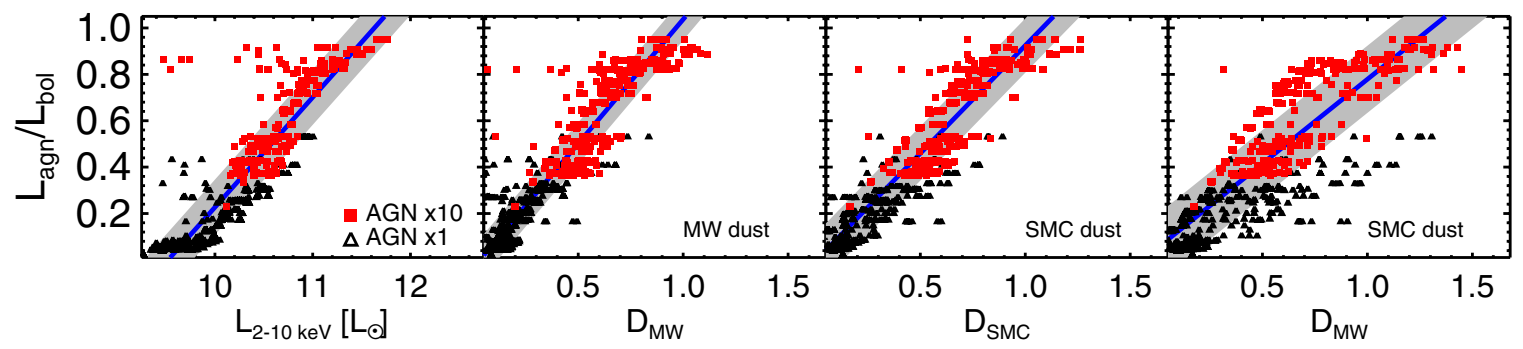

Figure 11. We show how diagnostic $D$ correlates with $L_{\mathrm{agn}} / L_{\mathrm{bol}}$ in our simulations in the right three panels. We show here only the highly obscured merger with our default ISM model, but the trends are similar for the alternate ISM model and the less obscured merger, as summarized in Table 3 . As a comparison, we plot the hard $\mathrm{X}$-ray flux through the 2-10 keV band in the left panel. If the correct dust model is used, then $D$ accurately predicts $L_{\mathrm{agn}} / L_{\mathrm{bol}}$ as well as $L_{2}-10 \mathrm{keV}$. In the rightmost panel, we apply $D_{\mathrm{Mw}}$ when the source has SMC dust instead, demonstrating that the scatter increases by $\sim 70 \%$. This issue can be mitigated by using, for example, Figure 10 to choose the appropriate indicator $D$.

(A color version of this figure is available in the online journal.)

Table 3

Ability to Predict $L_{\text {agn }} / L_{\text {bol }}$

\begin{tabular}{lcrcc}
\hline \hline Quantity, $Q^{\text {a }}$ & Scatter, $S^{\text {b }}$ & Intercept, $a$ & Slope, $b$ & Failure \% \\
\hline \multicolumn{5}{c}{ Default ISM } \\
\hline $\log L_{2-10 \mathrm{keV} / L_{\odot}}$ & 0.12 & -4.51 & 0.47 & 6.1 \\
$D_{\text {MW }}$, MW dust & 0.09 & -0.04 & 1.07 & 2.6 \\
$D_{\text {SMC }}$, SMC dust & 0.12 & -0.01 & 0.93 & 0.5 \\
$D_{\text {MW }}$, SMC dust & 0.14 & 0.05 & 0.72 & 0.8 \\
\hline \multicolumn{5}{c}{ Alternate ISM } \\
\hline $\log L_{2-10 \mathrm{keV} / L_{\odot}} 0.09$ & -4.59 & 0.47 & 0.8 \\
$D_{\text {MW }}$, MW dust & 0.10 & -0.04 & 0.96 & 0.9 \\
$D_{\text {SMC }}$, SMC dust & 0.09 & 0.00 & 0.86 & 0.0 \\
$D_{\text {MW }}$, SMC dust & 0.10 & 0.00 & 0.98 & 1.3 \\
\hline
\end{tabular}

Notes.

${ }^{\mathrm{a}} L_{\mathrm{agn}} / L_{\mathrm{bol}}=a+b(Q)$.

b $S=$ MAD/0.67, see Section 4.3.

c Percentage of points with $L_{\mathrm{agn}} / L_{\mathrm{bol}}$ further than $3 \sigma$ away from the fitted relation. In practice, for these cases the indicator predicts an $L_{\mathrm{agn}} / L_{\mathrm{bol}}$ smaller than the true value owing to extreme attenuation.

roughly the same median scatter, $S \sim 10 \%$. However, we find that $L_{2-10 \mathrm{keV}}$ experiences a failure rate $(>3 \sigma)$ two to three times higher than $D$ in the highly obscured calculation. Both quantities predict $L_{\mathrm{agn}} / L_{\mathrm{bol}}$ equally well under the alternate ISM treatment and for the less obscured candidate (not shown), with $S<10 \%$ and failure rates $<1 \%$.

Several simulated points at $L_{\text {agn }} / L_{\text {bol }} \approx 0.8$ fail in both $L_{2-10 \mathrm{kev}}$ and $D_{\mathrm{MW}}$. In these cases, corresponding to the most highly obscured lines of sight in our AGN $\times 10$ calculation from Figure 6, the mid-IR is so heavily attenuated that no $r_{9.7}$ signature from the central source survives in the observed SED (see also Section 4.3).

Diagnostic $D$ requires information about the galaxy's stellar mass (rest-frame $\lambda \sim 1-2 \mu \mathrm{m}$ ), limiting its immediate application to large new samples. However, the rest-frame wavelength range $D$ requires will be covered by the JWST, an observatory whose Mid-Infrared Instrument (MIRI; e.g., Wright et al. 2004) permits measurements of $r_{7.7}$ and $r_{9.7}$ for sources at $z \lesssim 1$. The tight correlation we find between $D$ and $L_{\text {agn }} / L_{\text {bol }}$ reflects the possible benefit in combining information about $r_{9.7}$ and $r_{7.7}$ with near-IR color diagnostics from JWST itself (e.g., Messias et al. 2012), or by matching to sources from other IR surveys (e.g., Stern et al. 2005, 2012). For the two example SEDs in Figure 12, whose known AGN fractions are both $L_{\mathrm{agn}} / L_{\mathrm{bol}}=0.33$, this procedure estimates

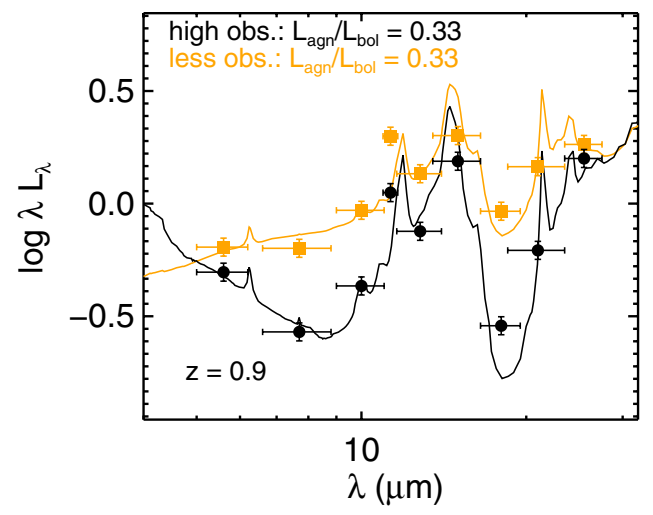

Figure 12. Synthetic photometric observations by MIRI on JWST, demonstrating the ability of future mid-IR technology to measure AGN power in systems with a wide range of obscuration. Here we assumed MW dust and $z=0.9$.

(A color version of this figure is available in the online journal.)

$L_{\text {agn }} / L_{\text {bol }}=0.33 \pm 0.09$ and $L_{\text {agn }} / L_{\text {bol }}=0.38 \pm 0.09$ (uncertainties are from the minimum theoretical scatter in the correlations) for the high and low obscuration examples, respectively.

\subsection{Limitations and Improvements}

A concern with the present method is that we cannot be sure how our few idealized simulations fit into a cosmological context. We have mitigated this issue by presenting two rather different ULIRG candidates, by varying the relative power between the AGNs and SF, and by varying the assumptions acting on dust obscuration and emission. We are planning to build a library of model SEDs for a much wider variety of systems, enabling more robust studies across IR-luminous galaxy populations. Moreover, we have focused here on AGN signatures at mid-infrared wavelengths, and information at longer wavelengths (cf. Younger et al. 2009), such as the location of the far-IR peak, could further constrain the AGN fractions in such sources (e.g., S12). In an upcoming paper (E. Roebuck et al., in preparation), we include the far-IR peak predictions from these simulations in interpreting the properties of $z \sim 0.3-3$ starbursts and quasars (the S12 sample).

The simplest interpretation of Figure 8 is that analogs of our "highly obscured" and "less obscured" examples are both realized in observed sources, in addition to both MW and SMC dust: all four models are required to cover the wide observed distributions of mid-IR color, PAH emission, and silicate absorption. A higher ratio of silicate to carbonaceous 
dust grains increases $r_{9.7}$ while shrinking $r_{7.7}$ (Figures 4), without changing $f_{4.5} / f_{1.6}$ (Figure 9), permitting estimates of $L_{\text {agn }} / L_{\text {bol }}$ under a variety of physical conditions, dust models, and optical depths. Although different ISM or dust models can produce broadly similar mid-IR trends, a separate measure of the AGN power, such as X-ray flux, is essential to further constrain these models. Furthermore, the recent discovery of high-redshift, hyper-luminous, hot-dust ULIRGs (Eisenhardt et al. 2012; Wu et al. 2012) suggests that we may lack a key phase in these few simulations, in which feedback initiates a transition between scenarios represented by our "highly obscured" and "less obscured" SED models.

Several assumptions regarding the dust RT may lead to discrepancies between our model sources and real sources. In particular, from Figure 8 we have identified two failures to which these assumptions likely contribute: (1) the mid-IR colors of the most AGN-dominated highly obscured sources are redder than their real counterparts (although bluer at $\lambda \lesssim 5 \mu \mathrm{m}$; see Figure 9), and (2) we do not simulate enough sources with little signs of PAH emission or silicate absorption: $r_{9.7} \sim 0$ and $r_{7.7} \sim 0$.

The IR calculation in SUNRISE distributes the energy absorbed by small PAH grains equally between emission in thermal equilibrium and in modes giving rise to the distinctive midIR PAH lines (Jonsson et al. 2010). Therefore, any change to this assumption or a more sophisticated calculation, such as thermally fluctuating small grains (e.g., Guhathakurta \& Draine 1989), will affect the dust continuum shape and PAH strength. In addition, we do not currently model dust destruction, and our model for dust production is crude, both key areas where nearfuture progress may be tenable. By not allowing our radiation to destroy dust grains or alter the dust grain distribution (or changing more generally the gas distribution owing to realistic feedback), we may be preventing ourselves from obtaining truly "power-law" AGN SEDs with no mid-IR dust features that may arise after AGN feedback disrupts the ISM.

We have purposefully focused on how galaxy dust can affect the fixed SMBH source SED (with minor variations in Section 4.4), rather than how the physics on smaller scales play out in observations of ULIRGs. While high levels of galaxy dust can reproduce a number of the properties of obscured quasars, the evolution of the central regions during these times of maximum obscuration will depend on the behavior of gas structures very close to the SMBH. This behavior may set the minimum scatter to be observed in correlations between these indicators. Using multiscale simulations, Hopkins et al. (2012a) showed how gravitational instabilities propagate down to scales of $\sim 1-100$ pc during large-scale gas inflows. They demonstrated that a number of properties of obscured AGNs attributed to a torus are explained by such dynamically-evolving accretion structures. Furthermore, these simulations suggest that the angular momentum vector of the small-scale "torus" can often be misaligned with the angular momentum of gas on galaxy scales, and the relative orientations can vary significantly in time (Hopkins et al. 2012b). Thus, the attenuation from the host galaxy may vary more significantly than our current simulations suggest. Therefore, we are motivated to develop methods of integrating knowledge of the impact of these different scales on the emergent SEDs as a means for further interpreting the evolution of IR-luminous galaxies.

Recent work on merger simulations at higher resolution (Hopkins et al. 2013) and with different hydrodynamics solvers (e.g., ArEPo: Springel 2010; C. Hayward et al., in prepara- tion) shows that the average global evolution in simulations like the ones we analyze here, and hence conclusions made based on them, are robust. However, such advances will enable more sophisticated treatments of feedback processes and hydrodynamics in gas with a complex phase structure and at smaller scales; therefore, future SED modeling could exploit these improvements to produce ever more detailed comparison libraries.

\section{SUMMARY AND CONCLUSIONS}

In this work, we used the detailed information contained in high-resolution hydrodynamical merger simulations to analyze the impact of galaxy-scale evolution of dust on interpretations of AGN power in ULIRGs derived from the mid-infrared. Using the dust RT code SUNRISE, we calculated the SED evolution during two model starbursts meant to represent possible extremes of observed ULIRG populations. One merger is characteristic of bright starbursts at $z \sim 2-4$, while the other is a typical gas-rich merger at $z \sim 0$.

Increasing computational resources will enable future analyses of many more galaxy and galaxy merger scenarios and more sophisticated modeling of the small-scale physics that are presently very uncertain. In this initial work, we studied the variation of the modeled mid-IR SEDs as a function of viewing angle, source SED, dust grain distribution, galaxy ISM clumpiness, and AGN power, and evaluated the ability of common mid-IR diagnostics to predict the AGN UV-IR luminosity fraction, $L_{\mathrm{agn}} / L_{\mathrm{bol}}$. We developed a possibly generic mid-IR estimator of $L_{\text {agn }} / L_{\text {bol }}$ that can be measured for new samples with next-generation instruments such as JWST.

Our main conclusions are:

1. Our modeling technique broadly reproduces the observed span of common mid-IR diagnostics used to disentangle AGN and starburst activity.

2. Although generally consistent with previous interpretations, none of the indicators straightforwardly predict $L_{\text {agn }} / L_{\text {bol }}$ because they depend non-linearly on the galaxy's properties, which can vary rapidly on timescales $\sim 10^{8} \mathrm{yr}$.

3. Highly obscured sources can be more AGN-dominated than realized, even when the shape of their SED resembles a pure starburst. A large enough column density reprocesses all of the direct AGN light into longer wavelengths.

4. The mid-IR SEDs of sources with a powerful, highly obscured AGN depend strongly on the direction from which the source is observed. Along some lines of sight, significant dust self-absorption attenuates the IR flux and obscures the mid-IR signatures of the buried AGN.

5. Sources with a deep $9.7 \mu \mathrm{m}$ silicate absorption feature can be reproduced by models either with an extremely luminous AGN and MW galaxy dust, or with an average-strength AGN and SMC galaxy dust.

6. It is possible to construct an idealized mid-IR indicator utilizing future JWST data to cleanly estimate $L_{\mathrm{agn}} / L_{\mathrm{bol}}$ while simultaneously constraining the dust grain model.

This research has made use of NASA's Astrophysics Data System. The computations in this paper were run on the Odyssey cluster supported by the FAS Science Division Research Computing Group at Harvard University. We thank the anonymous referee for valuable suggestions that improved this paper. 


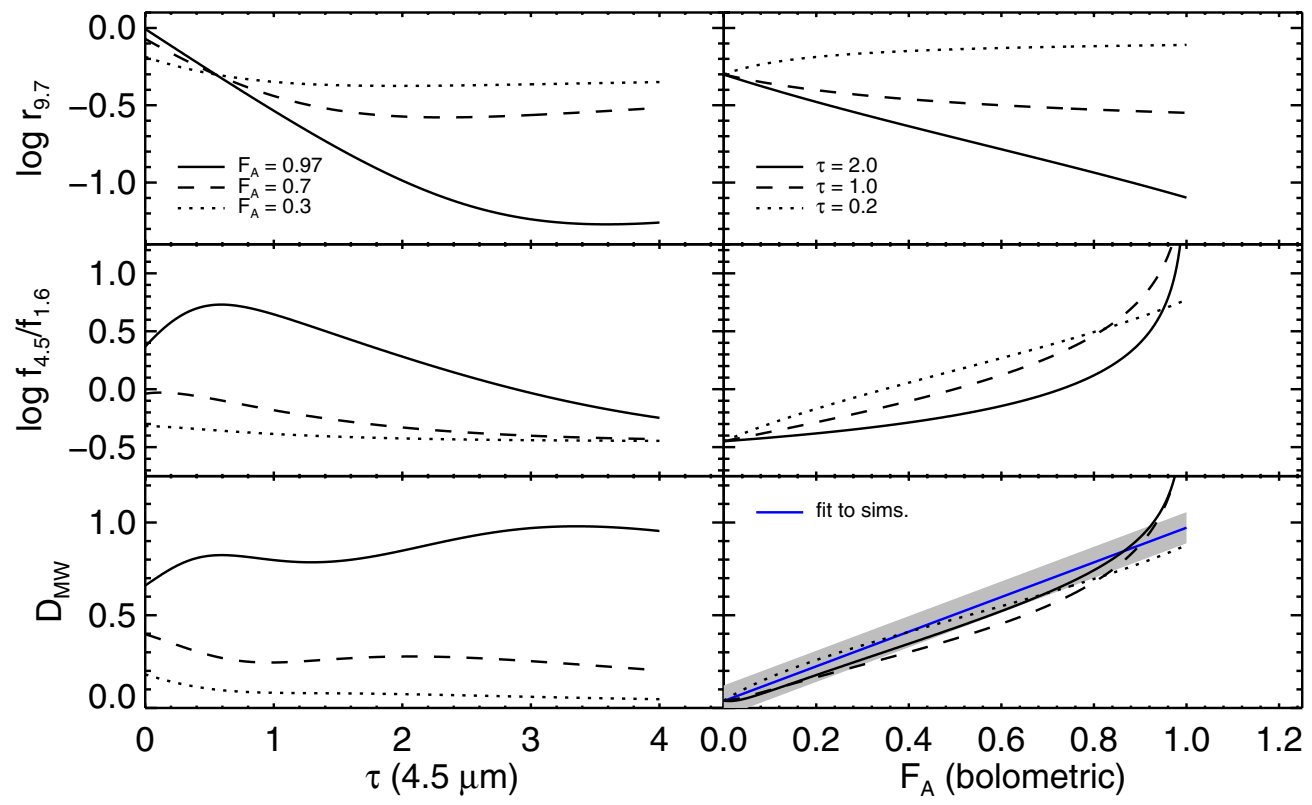

Figure 13. Behavior of toy models for $r_{9.7}, f_{4.5} / f_{1.6}$, and $D_{\mathrm{MW}}$ as a function of near-IR optical depth and AGN fraction. These simple models consist of an AGN source buried behind a simple obscuring screen ("galaxy dust"), plus an extended region of star formation that has a fixed spectral shape that does not depend on the optical depth to the AGN. In the upper left panel, $\log r_{9.7}$ has a strong dependence on $\tau$, even at low levels of obscuration, giving rise to the large viewing angle scatter of $r_{97}$ in the simulations of Section 4.3: since the AGN is a point source, its final SED depends only on attenuation along a single line of sight, which can vary widely, while indicators of activity over a larger spatial region (such as $r_{7.7}$ ) have their attenuation averaged over many lines of sight. Several other phenomena from the simulations are demonstrated by these models. (1) Generally, the silicate feature is enhanced as the AGN fraction increases and as $\tau$ increases, up until the SF gas begins to dominate the attenuated AGN SED, at which point the dependence of $r_{9.7}$ on $\tau$ reverses. (2) The near-IR color $f_{4.5} / f_{1.6}$ effectively selects powerful AGNs when $\tau$ is small enough (in our units, $\tau(4.5 \mu \mathrm{m}) \lesssim 1-2$ ). (3) At larger $\tau, \log f_{4.5} / f_{1.6}$ decreases and $\left|\log r_{9.7}\right|$ increases in such a way that the combination of these diagnostics, $D_{\mathrm{MW}}$, remains roughly constant for a fixed AGN fraction. This constancy is shown in the bottom left panel, and gives rise to the tight correlation between AGN fraction and $D_{\mathrm{MW}}$ in the bottom right panel. These simple arguments roughly verify the RT calculations and motivate the behavior of the empirically defined indicator $D_{\mathrm{MW}}$ (Section 6.1).

(A color version of this figure is available in the online journal.)

\section{APPENDIX}

\section{TOY MODELS FOR QUALITATIVE DIAGNOSTIC BEHAVIOR}

Here we make plausibility arguments based on a simple toy model to validate the simulated behavior of the diagnostics we analyzed. We assume a point source AGN that has an SED composed of direct AGN emission and dust re-emission from the torus. We label this flux $c_{\mathrm{A}}$. This is attenuated by a foreground screen such that the flux at infinity is $c_{\mathrm{A}} e^{-\tau}$. For the starburst component, $c_{\mathrm{S}}$, energy is absorbed by dust and re-emitted at IR wavelengths. Then, this dust emission is attenuated at larger scales with some effective optical depth.

The presence of silicate grains causes a sharp increase in the dust opacity near $\lambda=9.7 \mu \mathrm{m}$. Suppose that the attenuation curve is such that the attenuation on either side of the deep silicate feature is $e^{-\tau_{9.7}}$, but in the feature, the attenuation is $e^{-R \tau_{9.7}}$, where $R>1 . R$ represents the ratio of the peak of the full extinction curve at $9.7 \mu \mathrm{m}$ to that arising without the silicate absorption; here, we analyze $R=6$, which roughly matches the behavior we see in the simulations as expected with an extinction curve for MW-like dust (Li \& Draine 2001).

As in Section 3.1, $r_{9.7}=f_{9.7} / c_{9.7}$. We define the intrinsic monochromatic AGN fraction $F_{\mathrm{A}}=c_{\mathrm{A}} /\left(c_{\mathrm{A}}+c_{\mathrm{S}}\right)$. This quantity is closely related to, but not directly proportional to, the bolometric AGN fraction $L_{\mathrm{agn}} / L_{\mathrm{bol}}$ used throughout the rest of this paper and defined in Section 2.6. We estimate the conversion between these quantities in Equation (A4). By deriving toy diagnostics as a function of $F_{A}$, we can controllably analyze their behavior in much the same way that we explored the simulations in Sections 4-6 using the AGN $\times 0, A G N \times 1$, and AGN $\times 10$ models defined in Sections 2.3 and 2.4. Expanding $r_{9.7}$, we find

$$
r_{9.7}=\frac{f_{9.7}}{c_{9.7}}=\frac{c_{\mathrm{A}} e^{-R \tau_{9.7}}+a c_{\mathrm{S}}}{c_{\mathrm{A}} e^{-\tau_{9.7}}+c_{\mathrm{S}}}=\frac{F_{\mathrm{A}} e^{-R \tau_{9.7}}+a\left(1-F_{\mathrm{A}}\right)}{F_{\mathrm{A}} e^{-\tau_{9.7}}+\left(1-F_{\mathrm{A}}\right)},
$$

where $a$ is a new parameter that characterizes the silicate absorption feature imprinted on the IR energy emitted from SF. In the RT calculations, we obtain $a \sim 0.5$ since $\log _{10} r_{9.7} \sim$ -0.3 when $F_{\mathrm{A}} \sim 0$ (Figure 3 ); this corresponds to an effective optical depth to the star forming regions of $\tau_{9.7, \mathrm{SF}} \sim 0.13$. Since the SF gas is spatially extended, $\tau_{\mathrm{SF}}$ will change less with viewing angle than $\tau_{\mathrm{A}}$, allowing us to keep this parameter $a$ fixed.

The PAH fraction $r_{7.7}$ is defined to be $r_{7.7}=f_{7.7} / c_{7.7}$. Add new variables $p_{\mathrm{A}}$ and $p_{\mathrm{S}}$ for the PAH emission luminosity, and assume that the PAH emission is some fixed factor $P$ times the continuum emission for either AGN or SF. We expand to find

$$
\begin{aligned}
r_{7.7} & =\frac{\left(c_{\mathrm{A}}+p_{\mathrm{A}}\right) e^{-\tau_{7.7}}+\left(c_{\mathrm{S}}+p_{\mathrm{S}}\right)}{c_{\mathrm{A}} e^{-\tau_{7.7}}+c_{\mathrm{S}}} \\
& \approx \frac{(1+P) c_{\mathrm{A}} e^{-\tau_{7.7}}+(1+P) c_{\mathrm{S}}}{c_{\mathrm{A}} e^{-\tau_{7.7}}+c_{\mathrm{S}}} \sim 1+P .
\end{aligned}
$$

The true behavior is somewhat more complex than this, since in Figure 3 we see that $r_{7.7}$ varies quickly at high AGN 
fractions and obscuration levels, but since both the numerator and denominator have a similar dependence on $\tau_{7.7}$, there is minimal scatter with viewing angle at all times.

Now, if we ignore SF by setting $F_{\mathrm{A}}=1$, we get $r_{9.7}=$ $e^{-(R-1) \tau_{9.7}}$, or $\ln r_{9.7}=-(R-1) \tau_{9.7}$ for all $\tau_{9.7}$. The magnitude of this pre-factor $(R-1>1)$ implies that the depth of the silicate feature varies more strongly with optical depth, and thus viewing angle and time, than does the continuum. Its negative sign gives rise to the intuitive behavior that the silicate absorption feature becomes more apparent $\left(r_{9.7}\right.$ shrinks $)$ with increasing optical depth. However, this trend fails in, e.g., Figure 6, and the $D$ points scattered low in Figure 11.

For simplicity, in the case of mixed SF and AGN, let $a=0.5$ and $F_{\mathrm{A}}=0.9$, but let $\tau$ vary. When $\tau$ is very small, then the AGN terms dominate, yielding $\ln r_{9.7} \sim-(R-1) \tau, \tau \ll 1$, the same behavior as the no-SF case. When $\tau$ is large enough that the AGN term in the numerator of Equation (A1) can be safely ignored, then we have $r_{9.7} \approx 0.5 /\left(9 e^{-\tau}+1\right)$. This simplifies depending on $\tau$ :

$$
\ln r_{9.7} \approx-\ln 9+\tau, \quad \tau \sim 1, \quad \ln r_{9.7} \approx-9 e^{-\tau}, \quad \tau \gg 1
$$

Therefore, for powerful AGN, $r_{9.7}$ depends strongly on $\tau$, until $\tau$ gets too far above 1 , giving rise to the strong viewingangle scatter we noticed in the simulations in Section 4.3. In Figure 13, we plot example curves showing how $r_{9.7}$ and $r_{7.7}$ depend on $F_{\mathrm{A}}$ and $\tau$ in this toy model, which generally match the behavior we find in the simulations.

The qualitative behavior of diagnostic $D_{\mathrm{MW}}$ can be derived in the same fashion. For this, we seek a similar type of model for $f_{4.5} / f_{1.6}$. Roughly speaking (Figure 2), the pureAGN SED is such that $f_{4.5}=f_{1.6}\left(v_{1.6} / v_{4.5}\right)=2.8 f_{1.6}$, and the stellar SED is $f_{4.5}=f_{1.6}\left(v_{4.5} / v_{1.6}\right)=f_{1.6} / 2.8$. The added complication in this situation is that the attenuation of the AGN source depends on wavelength, roughly speaking as $\tau(\lambda) \sim \tau(1 \mu \mathrm{m}) \times(\lambda / 1 \mu \mathrm{m})^{1.5}$ (e.g., Weingartner \& Draine $2001)$. We can use the same correction when combining $r_{9.7}$ and $f_{4.5} / f_{1.6}$ to model the $D_{\mathrm{MW}}$ diagnostic. We get

$$
\frac{f_{4.5}}{f_{1.6}} \approx \frac{F_{\mathrm{A}} e^{-\tau_{4.5}}+\left(1-F_{\mathrm{A}}\right)}{\left(F_{\mathrm{A}} / 2.8\right) e^{-\tau_{4.5} 2.8^{1.5}}+2.8\left(1-F_{\mathrm{A}}\right)} .
$$

In Figure 13, we construct $f_{4.5} / f_{1.6}$ and $D_{\mathrm{MW}}$ analytically and plot their behavior as a function of $F_{\mathrm{A}}$ and $\tau$ (at $4.5 \mu \mathrm{m}$ ). A final difference between the simulated quantities and these toy models is that we derived these models as a function of a monochromatic AGN fraction, while the quantity $L_{\mathrm{agn}} / L_{\mathrm{bol}}$ used in the rest of this paper is the bolometric AGN fraction. The correction depends on the SED shape of the various components, which we parameterize as $P_{\mathrm{A}}=j P_{\mathrm{A}, \text { bol }}, P_{\mathrm{SF}}=k P_{\mathrm{SF}, \text { bol }}$. The AGN bolometric correction factor $j$ is fixed since our input template has a fixed SED shape over the wavelengths used by SUNRISE, while the SF correction $k$ varies; we find that $j / k \approx 5 \pm 2$ in our RT calculations, and so we derive

$$
\begin{aligned}
F_{\mathrm{A}} & =\frac{P_{\mathrm{A}}}{P_{\mathrm{A}}+P_{\mathrm{SF}}}=\frac{k P_{\mathrm{A}, \text { bol }}}{k P_{\mathrm{A}, \text { bol }}+j P_{\mathrm{SF}, \text { bol }}} \\
& =\frac{(k / j) F_{\mathrm{A}, \text { bol }}}{F_{\mathrm{A}, \text { bol }}\left(\frac{k}{j}-1\right)+1} \sim \frac{5 F_{\mathrm{A}, \text { bol }}}{4 F_{\mathrm{A}, \text { bol }}+1} .
\end{aligned}
$$

Figure 13 shows $r_{9.7}, f_{4.5} / f_{1.6}$, and $D_{\mathrm{MW}}$ from these idealized formulae for a sampling of AGN fractions and optical depths, confirming much of the behavior we observed in the results of the RT calculations.

\section{REFERENCES}

Armus, L., Bernard-Salas, J., Spoon, H. W. W., et al. 2006, ApJ, 640, 204 Armus, L., Charmandaris, V., Bernard-Salas, J., et al. 2007, ApJ, 656, 148 Bauer, F., Yan, L., Sajina, A., \& Alexander, D. 2010, ApJ, 710, 212

Beers, T., Flynn, K., \& Gebhardt, K. 1990, AJ, 100, 32

Bouwens, R. J., Illingworth, G. D., Franx, M., \& Ford, H. 2007, ApJ, 670, 928 Brandl, B., Bernard-Salas, J., Spoon, H. W. W., et al. 2006, ApJ, 653, 1129

Bush, S. J., Cox, T. J., Hayward, C. C., et al. 2010, ApJ, 713, 780

Caputi, K., Lagache, G., Yan, L., et al. 2007, ApJ, 660, 97

Chakrabarti, S., Cox, T. J., Hernquist, L., et al. 2007, ApJ, 658, 840

Cox, T., Dutta, S. N., Matteo, T. D., et al. 2006a, ApJ, 650, 791

Cox, T., Jonsson, P., Primack, J., \& Somerville, R. 2006b, MNRAS, 373, 1013

Dale, D., Gil de Paz, A., Gordon, K. D., et al. 2007, ApJ, 655, 863

Dale, D., Smith, J. D. T., Armus, L., et al. 2006, ApJ, 646, 161

Dasyra, K. M., Yan, L., Helou, G., et al. 2009, ApJ, 701, 1123

Di Matteo, T., Springel, V., \& Hernquist, L. 2005, Natur, 433, 604

Dole, H., Lagache, G., Puget, J.-L., et al. 2006, A\&A, 451, 417

Dopita, M., Groves, B. A., Fischera, J., et al. 2005, ApJ, 619, 755

Draine, B. T., Dale, D. A., Bendo, G., et al. 2007, ApJ, 663, 866

Draine, B. T., \& Li, A. 2001, ApJ, 551, 807

Draine, B. T., \& Li, A. 2007, ApJ, 657, 810

Dwek, E. 1998, ApJ, 501, 643

Eisenhardt, P. R. M., Wu, J., Tsai, C.-W., et al. 2012, ApJ, 755, 173

Fadda, D., Yan, L., Lagache, G., et al. 2010, ApJ, 719, 425

Faucher-Giguère, C.-A., Lidz, A., Zaldarriaga, M., \& Hernquist, L. 2009, ApJ, 703,1416

Fazio, G., Hora, J. L., Allen, L. E., et al. 2004, ApJS, 154, 10

Ferrarese, L., \& Merritt, D. 2000, ApJL, 539, L9

Genzel, R., Lutz, D., Sturm, E., et al. 1998, ApJ, 498, 579

Georgantopoulos, I., Dasyra, K. M., Rovilos, E., et al. 2011, A\&A, 531, A116

Gordon, K. D., Calzetti, D., \& Witt, A. N. 1997, ApJ, 487, 625

Gordon, K., \& Clayton, G. 1998, ApJ, 500, 816

Gordon, K. D., Clayton, G. C., Misselt, K. A., Landolt, A. U., \& Wolff, M. J. 2003, ApJ, 594, 279

Groves, B., Dopita, M., Sutherland, R., et al. 2008, ApJS, 176, 438

Guhathakurta, P., \& Draine, B. T. 1989, ApJ, 345, 230

Gültekin, K., Richstone, D. O., Gebhardt, K., et al. 2009, ApJ, 698, 198

Hayward, C. C., Jonsson, P., Kereš, D., et al. 2012, MNRAS, 424, 951

Hayward, C., Kereš, D., Jonsson, P., et al. 2011, ApJ, 743, 159

Hayward, C. C., Narayanan, D., Keres, D., et al. 2013, MNRAS, 428, 2529

Hernquist, L. 1990, ApJ, 356, 359

Hernquist, L., \& Katz, N. 1989, ApJS, 70, 419

Hickox, R. C., Wardlow, J. L., Smail, I., et al. 2012, MNRAS, 421, 284

Hönig, S. F., Beckert, T., Ohnaka, K., \& Weigelt, G. 2006, A\&A, 452, 459

Hönig, S. F., Kishimoto, M., Gandhi, P., et al. 2010, A\&A, 515, A23

Hopkins, P. F., Cox, T. J., Hernquist, L., et al. 2013, MNRAS, 1, 733

Hopkins, P. F., Hayward, C. C., Narayanan, D., \& Hernquist, L. 2012a, MNRAS, 420,320

Hopkins, P., Hernquist, L., Cox, T., Robertson, B., \& Krause, E. 2007a, ApJ, 669,45

Hopkins, P., Hernquist, L., Cox, T., Robertson, B., \& Krause, E. 2007b, ApJ, 669,67

Hopkins, P., Hernquist, L., Cox, T., et al. 2005, ApJ, 630, 705

Hopkins, P., Hernquist, L., Cox, T., et al. 2006, ApJS, 163, 1

Hopkins, P. F., Hernquist, L., Hayward, C. C., \& Narayanan, D. 2012b, MNRAS, 425,1121

Hopkins, P., Richards, G. T., \& Hernquist, L. 2007c, ApJ, 654, 731

Hopkins, P. F., Strauss, M. A., Hall, P. B., et al. 2004, AJ, 128, 1112

Hopkins, P., Younger, J. D., Hayward, C. C., Narayanan, D., \& Hernquist, L. 2010, MNRAS, 402, 1693

Houck, J., Roellig, T. L., Van Cleve, J., et al. 2004, Proc. SPIE, 5487, 62

Houck, J., Soifer, B. T., Weedman, D., et al. 2005, ApJL, 622, L105

Imanishi, M., Nakagawa, T., Ohyama, Y., et al. 2008, PASJ, 60, 489

Johnson, H., \& Morgan, W. 1953, ApJ, 117, 313

Jonsson, P. 2006, MNRAS, 372, 2

Jonsson, P., Groves, B. A., \& Cox, T. J. 2010, MNRAS, 403, 17

Juneau, S., Dickinson, M., Alexander, D. M., \& Salim, S. 2011, ApJ, 736, 104

Katz, N., Weinberg, D. H., \& Hernquist, L. 1996, ApJS, 105, 19

Kennicutt, R. C. 1998, ApJ, 498, 541

Kormendy, J., \& Richstone, D. 1995, ARA\&A, 33, 581

Lacy, M., Sajina, A., Petric, A. O., et al. 2007, ApJ, 669, L61

Laor, A., \& Draine, B. T. 1993, ApJ, 402, 441

Laurent, O., Mirabel, I., Charmandaris, V., et al. 2000, A\&A, 359, 887

Le Floc'h, E., Papovich, C., Dole, H., et al. 2005, ApJ, 632, 169

Leitherer, C., Schaerer, D., Goldader, J. D., et al. 1999, ApJS, 123, 3 
Levenson, N., Heckman, T., Krolik, J., Weaver, K., \& Życki, P. 2006, ApJ, 648,111

Levenson, N., Sirocky, M., Hao, L., et al. 2007, ApJL, 654, L45

Li, A., \& Draine, B. T. 2001, ApJ, 554, 778

Lutz, D., Genzel, R., Sternberg, A., et al. 1996, A\&A, 315, L137

Lutz, D., Maiolino, R., Spoon, H., \& Moorwood, A. 2004, A\&A, 418, 465

Lutz, D., Spoon, H., Rigopoulou, D., Moorwood, A., \& Genzel, R. 1998, ApJL, 505, L103

Magorrian, J., Tremaine, S., Richstone, D., et al. 1998, AJ, 115, 2285

Messias, H., Afonso, J., Salvato, M., Mobasher, B., \& Hopkins, A. 2012, ApJ, 754,120

Morrison, R., \& McCammon, D. 1983, ApJ, 270, 119

Mosteller, F., \& Tukey, J. 1977, Data Analysis and Regression. A Second Course in Statistics (Reading, MA: Addison-Wesley)

Narayanan, D., Dey, A., Hayward, C. C., et al. 2010a, MNRAS, 407, 1701

Narayanan, D., Hayward, C. C., Cox, T. J., et al. 2010b, MNRAS, 401, 1613

Nenkova, M., Sirocky, M., Nikutta, R., Ivezić, v., \& Elitzur, M. 2008, ApJ, 685,160

Pier, E., \& Krolik, J. 1992, ApJ, 401, 99

Pope, A., Bussmann, R. S., Dey, A., et al. 2008, ApJ, 689, 127

Richards, G., Lacy, M., Storrie-Lombardi, L. J., et al. 2006a, ApJS, 166, 470

Richards, G. T., Strauss, M. A., Fan, X., et al. 2006b, AJ, 131, 2766

Rigopoulou, D., Spoon, H., Genzel, R., et al. 1999, AJ, 118, 2625

Robertson, B., Hernquist, L., Cox, T. J., et al. 2006, ApJ, 641, 90

Roussel, H., Helou, G., Beck, R., et al. 2003, ApJ, 593, 733

Sajina, A., Spoon, H., Yan, L., et al. 2009, ApJ, 703, 270

Sajina, A., Yan, L., Fadda, D., Dasyra, K., \& Huynh, M. 2012, ApJ, 757, 13

Sajina, A., Yan, L., Lacy, M., \& Huynh, M. 2007, ApJ, 667, L17

Sanders, D., \& Mirabel, I. 1996, ARA\&A, 34, 749

Sanders, D., Soifer, B., Elias, J., et al. 1988, ApJ, 325, 74

Schweitzer, M., Lutz, D., Sturm, E., et al. 2006, ApJ, 649, 79
Siebenmorgen, R., Haas, M., Krügel, E., \& Schulz, B. 2005, A\&A, 436, L5 Silk, J., \& Rees, M. 1998, A\&A, 331, L1

Smith, J., Draine, B. T., Dale, D. A., et al. 2007, ApJ, 656, 770

Snyder, G., Cox, T., Hayward, C., Hernquist, L., \& Jonsson, P. 2011, ApJ, 741, 77

Spoon, H., Marshall, J., Houck, J., et al. 2007, ApJL, 654, L49

Springel, V. 2005, MNRAS, 364, 1105

Springel, V. 2010, MNRAS, 401, 791

Springel, V., Di Matteo, T., \& Hernquist, L. 2005, MNRAS, 361, 776

Springel, V., \& Hernquist, L. 2002, MNRAS, 333, 649

Springel, V., \& Hernquist, L. 2003, MNRAS, 339, 289

Stern, D., Assef, R. J., Benford, D. J., et al. 2012, ApJ, 753, 30

Stern, D., Eisenhardt, P., Gorjian, V., et al. 2005, ApJ, 631, 163

Sturm, E., Lutz, D., Tran, D., et al. 2000, A\&A, 358, 481

Sturm, E., Rupke, D., Contursi, A., et al. 2006, ApJL, 653, L13

Surace, J. A., \& Sanders, D. B. 1999, ApJ, 512, 162

Surace, J. A., Sanders, D. B., \& Evans, A. S. 2000, ApJ, 529, 170

Tacconi, L. J., Genzel, R., Neri, R., et al. 2010, Natur, 463, 781

Tran, Q., Lutz, D., Genzel, R., et al. 2001, ApJ, 552, 527

Tremaine, S., Gebhardt, K., Bender, R., et al. 2002, ApJ, 574, 740

Veilleux, S., Rupke, D. S. N., Kim, D.-C., et al. 2009, ApJS, 182, 628

Vijh, U. P., Witt, A. N., \& Gordon, K. D. 2003, ApJ, 587, 533

Weingartner, J. C., \& Draine, B. T. 2001, ApJ, 548, 296

Werner, M., Roellig, T. L., Low, F. J., et al. 2004, ApJS, 154, 1

Witt, A. N., Thronson, H. A., \& Capuano, J. M. 1992, ApJ, 393, 611

Wright, G., Rieke, G. H., Colina, L., et al. 2004, Proc. SPIE, 5487, 653

Wu, J., Tsai, C.-W., Sayers, J., et al. 2012, ApJ, 756, 96

Wu, Y., Charmandaris, V., Hao, L., et al. 2006, ApJ, 639, 157

Wuyts, S., Cox, T. J., Hayward, C. C., et al. 2010, ApJ, 722, 1666

Yan, L., Sajina, A., Fadda, D., et al. 2007, ApJ, 658, 778

Younger, J., Hayward, C. C., Narayanan, D., et al. 2009, MNRAS, 396, L66 